\title{
WestVirginiaUniversity
}

THE RESEARCH REPOSITORY @ WVU

Graduate Theses, Dissertations, and Problem Reports

2016

\section{A study on Dicycles and Eulerian Subdigraphs}

\author{
Khalid A. Alsatami
}

Follow this and additional works at: https://researchrepository.wvu.edu/etd

\section{Recommended Citation}

Alsatami, Khalid A., "A study on Dicycles and Eulerian Subdigraphs" (2016). Graduate Theses,

Dissertations, and Problem Reports. 5088.

https://researchrepository.wvu.edu/etd/5088

This Dissertation is protected by copyright and/or related rights. It has been brought to you by the The Research Repository @ WVU with permission from the rights-holder(s). You are free to use this Dissertation in any way that is permitted by the copyright and related rights legislation that applies to your use. For other uses you must obtain permission from the rights-holder(s) directly, unless additional rights are indicated by a Creative Commons license in the record and/ or on the work itself. This Dissertation has been accepted for inclusion in WVU Graduate Theses, Dissertations, and Problem Reports collection by an authorized administrator of The Research Repository @ WVU.

For more information, please contact researchrepository@mail.wvu.edu. 


\title{
A study on Dicycles and Eulerian Subdigraphs
}

\author{
Khalid A. Alsatami \\ Dissertation submitted to the \\ Eberly College of Arts and Sciences \\ at West Virginia University \\ in partial fulfillment of the requirements \\ for the degree of \\ Doctor of Philosophy \\ in \\ Mathematics \\ Hong-Jian Lai, Ph.D., Chair \\ John Goldwasser, Ph.D. \\ Rong Luo, Ph.D. \\ K. Subramani, Ph.D. \\ Jerzy Wojciechowski, Ph.D. \\ Department of Mathematics \\ West Virginia University \\ Morgantown, West Virginia 26506 \\ 2016
}

Key words: Eulerian Digraphs, Supereulerian Digraphs, Local Structures,Symmetrically Connected Digraphs, Partially Symmetric Digraphs, 2-sums of Digraphs, Dicycles cover of Digraphs.

\section{Copyright 2016 Khalid A. Alsatami}




\section{ABSTRACT \\ A study on Dicycles and Eulerian Subdigraphs}

\section{Khalid A. Alsatami}

A dicycle cover of a digraph $D$ is a collection $\mathcal{C}$ of dicycles of $D$ such that $\bigcup_{C \in \mathcal{C}} A(C)=$ $A(D)$. If $D$ is obtained from a simple undirected graph $G$ by assigning an orientation to the edges of $G$, then $D$ is an oriented graph. A digraph $\mathrm{D}$ is eulerian if $\mathrm{D}$ is connected and for any $v \in V(D), d_{D}^{+}(v)=d_{D}^{-}(v)$. A digraph $D$ is supereulerian if $D$ contains a spanning eulerian subdigraph. A digraph $D$ is a closed ditrail if it is eulerian. This dissertation focuses on a study of dicycle cover and supereulerian digraphs from the following aspects.

1. Dicycle cover of Hamiltonian oriented graphs.

A dicycle cover of a digraph $D$ is a family $\mathcal{F}$ of dicycles of $D$ such that each arc of $D$ lies in at least one dicycle in $\mathcal{F}$. We investigate the problem of determining the upper bounds for the minimum number of dicycles which cover all arcs in a strong digraph. Best possible upper bounds of dicycle covers are obtained in a number of classes of digraphs, including strong tournaments, Hamiltonian oriented graphs, Hamiltonian oriented complete bipartite graphs, and families of possibly non-hamiltonian digraphs obtained from these digraphs via a sequence of 2 -sum operations.

2. Supereulerian digraphs with given local structures .

Catlin in 1988 indicated that there exist graph families $\mathcal{F}$ such that if every edge $e$ in a graph $G$ lies in a subgraph $H_{e}$ of $G$ isomorphic to a member in $\mathcal{F}$, then $G$ is supereulerian. In particular, if every edge of a connected graph $G$ lies in a 3-cycle, then $G$ is supereulerian. The purpose of this research is to investigate how Catlin's theorem can be extended to digraphs. A strong digraph $D$ is supereulerian if $D$ contains a spanning eulerian subdigraph. We show that there exists an infinite family of non-supereulerian strong digraphs each arc of which lies in a directed 3 -cycle. We also show that there exist digraph families $\mathcal{H}$ such that a strong digraph $D$ is supereulerian if every arc $a$ of $D$ lies in a subdigraph $H_{a}$ isomorphic to a member of $\mathcal{H}$. A digraph $D$ is symmetric if $(x, y) \in A(D)$ implies $(y, x) \in A(D)$; and is symmetrically connected if every pair of vertices of $D$ are joined by a symmetric dipath. A digraph $D$ is partially symmetric if the digraph obtained from $D$ by contracting all symmetrically connected components is symmetrically connected. It is known that a partially symmetric digraph may not be symmetrically 
connected. We show that symmetrically connected digraphs and partially symmetric digraphs are such families. Sharpness of these results are discussed.

3. On a class of supereulerian digraphs.

The 2-sum of two digraphs $D_{1}$ and $D_{2}$, denoted $D_{1} \oplus_{2} D_{2}$, is the digraph obtained from the disjoint union of $D_{1}$ and $D_{2}$ by identifying an arc in $D_{1}$ with an $\operatorname{arc}$ in $D_{2}$. A digraph $D$ is supereulerian if $D$ contains a spanning eulerian subdigraph. It has been noted that the 2 -sum of two supereulerian (or even hamiltonian) digraphs may not be supereulerian. We obtain several sufficient conditions on $D_{1}$ and $D_{2}$ for $D_{1} \oplus_{2} D_{2}$ to be supereulerian. In particular, we show that if $D_{1}$ and $D_{2}$ are symmetrically connected or partially symmetric, then $D_{1} \oplus_{2} D_{2}$ is supereulerian. 


\section{Acknowledgements}

First and foremost, I am most indebted to my supervisor, Professor Hong-Jian Lai, for his continued encouragement and support over these last few years. It is a pleasure to work under his supervision. Without of Allah then him, this paper could not have come about.

I would also like to thank my other committee members: Professor John Goldwasser, Professor Rong Luo,Professor K. Subramani and Professor Jerzy Wojciechowski for their help during my studies.

And finally, I would like to thank the Department of Mathematics and Eberly College of Arts

and Sciences at West Virginia University for providing me with an excellent study environment and support during my study as a graduate student. 


\section{DEDICATION}

To

my father $\underline{\text { Ali }}$, my mother $\underline{\text { Fatema }}$, my wife $\underline{\text { Hanan }}$

and

my lovely children Ali, Abdulkreem, Azam and Haitham 


\section{Contents}

1 Preliminaries $\quad 1$

1.1 Notation and Terminology . . . . . . . . . . . . . . . . . . 1

1.2 Previous Work On Supereulerian Digraphs . . . . . . . . . . . . . . . 4

1.3 Main Results . . . . . . . . . . . . . . . . . . . . . . . 7

2 Dicycle cover of Hamiltonian oriented graphs $\quad 9$

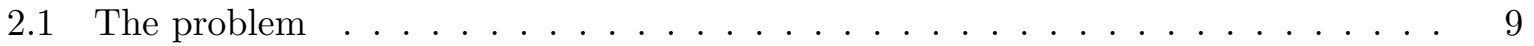

2.2 Proof of the Main Result _ . . . . . . . . . . . . . . . . . . . . . 10

2.2 .1 Tournament . . . . . . . . . . . . . . . . 16

$2.2 .2 \quad$ A Complete Bipartite . . . . . . . . . . . . . . . . . . 17

2.3 Dicycle Covers of $2-$ Sums of Digraphs _ . . . . . . . . . . . . . . . . . 20

3 Supereulerian Digraphs with given local structures $\quad 24$

3.1 Introduction . . . . . . . . . . . . . . . . . . . 24

3.2 Examples of nonsupereulerian strong digraphs . . . . . . . . . . . . . 26

3.3 Locally symmetrically connected supereulerian digraphs $\ldots \ldots \ldots$

3.4 Partially symmetric supereulerian digraphs f . . . . . . . . . . . . . . . 30 
3.5 Locally $k$-Eulerian Digraphs $\ldots \ldots \ldots \ldots \ldots \ldots$

3.6 A k-free trailable Digraphs . . . . . . . . . . . . . . . . . . 39

4 On a class of supereulerian digraphs $\quad 43$

4.1 Introduction . . . . . . . . . . . . . . . . . 43

4.2 The 2 -sums of digraphs $\ldots \ldots \ldots \ldots \ldots$

4.3 Sufficient conditions for supereulerian 2 -sums of digraphs $\ldots \ldots \ldots 7$ 


\section{Chapter 1}

\section{Preliminaries}

\section{$1.1 \quad$ Notation and Terminology}

We consider finite and simple graphs and digraphs. A digraph $D$ is simple if $D$ has no loops and if for any pair of distinct vertices $u, v \in V(D)$, there is at most one arc in $D$ oriented from $u$ to $v$. Usually, we use $G$ to denote a graph and $D$ a digraph. Undefined terms and notations will follow [1] for graphs and [2] for digraphs. In particular, $\kappa(G), \kappa^{\prime}(G)$ and $\alpha(G)$ denote the connectivity, the edge connectivity and the independence number of a graph $G$; and $\kappa(D)$ and $\lambda(D)$ denotes the vertex-strong connectivity and the arc-strong connectivity of a digraph $D$, respectively. In this chapter we will provide most of the terminology and notation used in this dissertation.

A cycle is a 2-regular connected nontrivial graph. A cycle on $n$ vertices is often called an $n$-cycle. A cycle cover of a graph $G$ is a collection $\mathcal{C}$ of cycles of $G$ such that $E(G)=\bigcup_{C \in \mathcal{C}} E(C)$. Bondy [4] conjectured that if $G$ is a 2-connected simple graph with $n \geq 3$ vertices, then $G$ has a cycle cover $\mathcal{C}$ with $|\mathcal{C}| \leq \frac{2 n-3}{3}$. Bondy [4] showed that this conjecture, if proved, would be best possible. Y. X. Luo and R. S. Chen [14] proved that this conjecture holds for 2-connected simple cubic graphs. It has been shown that for plane triangulations, serial-parallel graphs or planar graphs in general, one can have a better bound for the number of cycles used in a cover ( [9-12]). Barnette [3] proved that if $G$ is a 3 -connected simple planar graph of order $n$, then

the edges of $G$ can be covered by at most $\frac{n+1}{2}$ cycles. Fan [6] settled this conjecture by showing that it holds for all simple 2-connected graphs. The best possible number of cycles needed to cover cubic graphs has been obtained in [13] and [15]. 
we use the notation $(u, v)$ to denote an arc oriented from $u$ to $v$ in a digraph. A directed path in a digraph $D$ from a vertex $u$ to a vertex $v$ is called a $(u, v)$-dipath. To emphasize the distinction between graphs and digraphs, a directed cycle or path in a digraph is often referred as a dicycle or dipath. It is natural to consider the number of dicycles needed to cover a digraph. For a digraph $D, V(D)$ and $A(D)$ denote the vertex set and arc set of $D$, respectively. If $A^{\prime} \subseteq A(D)$, then $D\left[A^{\prime}\right]$ is the subdigraph induced by $A^{\prime}$. For an integer $n>0$, we use $K_{n}^{*}$ to denote the complete digraph on $n$ vertices. Hence for every pair of distinct vertices $u, v \in V\left(K_{n}^{*}\right)$, there is exactly one arc $(u, v)$ in $A\left(K_{n}^{*}\right)$. Any simple digraph $D$ on $n$ vertices can be viewed as a subdigraph of $K_{n}^{*}$. If $W$ is an arc subset of $A\left(K_{n}^{*}\right)$, then $D+W$ denotes the digraph $K_{n}^{*}[A(D) \cup W]$. If $(u, v)$ is an arc, we say that $u$ dominates $v$ or $(v$ is dominated by $u)$ and denote it by $u \rightarrow v$. For disjoint subsets $X$ and $Y$ of $V(D), X \rightarrow Y$ means that every vertex of $X$ dominates every vertex of $Y$. A digraph $D$ is strong if for any distinct $u, v \in V(D), D$ has a $(u, v)$-dipath. Following [2], if $X, Y \subseteq V(D)$, then define

$$
(X, Y)_{D}=\{(x, y) \in A(D): x \in X, y \in Y\} .
$$

When $Y=V(D)-X$, we define

$$
\partial_{D}^{+}(X)=(X, V(D)-X)_{D} \text { and } \partial_{D}^{-}(X)=(V(D)-X, X)_{D}
$$

If $X \subseteq V(D) \cup A(D)$, then $D[X]$ denotes the subdigraph induced by $X$. If $S$ is a subdigraph of a digraph $D$ and if $X \subset A(S)$ and $Y \subseteq A(D)-A(S)$, we use $S-X+Y$ to denote $D[(A(S)-X) \cup Y]$. For a vertex $v \in V(D)$, the out-degree of $v$ is $d_{D}^{+}(v)=\left|\partial_{D}^{+}(\{v\})\right|$ and the in-degree of $v$ in $D$ is $d_{D}^{-}(v)=\left|\partial_{D}^{-}(\{v\})\right|$. Furthermore, we define

$$
\partial_{D}(v)=\partial_{D}^{+}(\{v\}) \cup \partial_{D}^{-}(\{v\}) \text { and } d_{D}(v)=d_{D}^{+}(v)+d_{D}^{-}(v) .
$$

Let $N_{D}^{+}(v)=\{u \in V(D):(v, u) \in A(D)\}$ and $N_{D}^{-}(v)=\{u \in V(D):(u, v) \in A(D)\}$ denote the out neighbors and in neighbors of $v$ in $D$, respectively. When the digraph $D$ is understood from the context, we often omit the subscript $D$. Following [1] and [2], we use $\lambda(D)$ and $c(D)$ to denote the arc-strong connectivity of $D$ and the number of components of the underlying graph $G(D)$ of $D$, respectively. By the definition of $\lambda(D)$ in [2], for any integer $k \geq 0$,

$$
\lambda(D) \geq k \text { if and only if for any nonempty proper subset } X \subset V(D),\left|\partial_{D}^{+}(X)\right| \geq k .
$$

A dicycle cover of a digraph $D$ is a collection $\mathcal{C}$ of dicycles of $D$ such that $\bigcup_{C \in \mathcal{C}} A(C)=$ $A(D)$. If $D$ is obtained from a simple undirected graph $G$ by assigning an orientation to the edges of $G$, then $D$ is an oriented graph.

If $D$ is a digraph, we often use $G(D)$ to denote the underlying undirected graph of $D$, the graph obtained from $D$ by erasing all orientation on the $\operatorname{arcs}$ of $D$. 
For graphs $H$ and $G$, by $H \subseteq G$ we mean that $H$ is a subgraph of $G$. Similarly, for digraphs $H$ and $D$, by $H \subseteq D$ we mean that $H$ is a subdigraph of $D$.

For an integer $n$, we use the notation $[n]$ to denote the set $\{1,2, \ldots, n\}$. A walk in $D$ is an alternating sequence $W=x_{1} a_{1} x_{2} a_{2} x_{3} \cdots x_{k-1} a_{k-1} x_{k}$ of vertices $x_{i}$ and $\operatorname{arcs} a_{j}$ from $D$ such that the tail of $a_{j}$ is $x_{j}$ and the head of $a_{j}$ is $x_{j+1}$ for every $i \in[k]$ and $j \in[k-1]$. A walk $W$ is closed if $x_{1}=x_{k}$, and open otherwise. The set of vertices $\left\{x_{i}: i \in[k]\right\}$ is denoted by $V(W)$; the set of $\operatorname{arcs}\left\{a_{j}: j \in[k-1]\right\}$ is denoted by $A(W)$.

Following the definition in [2] (Page 11), for a subdigraph $H$ of a digraph $D$, an $(x, y)$-dipath $P$ is an $(H, H)$-dipath if $x, y \in V(H)$ and $V(P) \cap V(H)=\{x, y\}$.

A ditrail in $D$ is an alternating sequence of vertices and arcs such that all the arcs are distinct. If all the vertices of a ditrail are distinct we call it a dipath. An $\operatorname{arc}(u, v) \in A(D)$ is symmetric in $D$ if $(u, v),(v, u) \in A(D)$. A digraph $D$ is symmetric if $|V(D)|=1$ or if $|A(D)|>0$ and every arc of $D$ is symmetric. Especially, a symmetric dipath $P$ is a dipath such that every arc of $P$ is symmetric.

A digraph $D$ is trialable if there exist $x, y \in V(D)$, such that $D$ has a spanning $(x, y)$ ditrail.

A dipath $P$ is a hamiltonian dipath if $V(P)=V(D)$. A digraph $D$ is hamiltonian if $D$ contains a hamiltonian dicycle. An $(x, y)$-hamiltonian dipath is a hamiltonian dipath from $x$ to $y$. A digraph $D$ is hamiltonian-connected if $D$ has an $(x, y)$-hamiltonian dipath for every choice of distinct vertices $x, y \in V(D)$.

A digraph $D$ is weakly connected if $G(D)$, its underlying graph, is connected. Let $\mathcal{H}$ be a family of digraphs. A strong digraph $D$ is locally $\mathcal{H}$ if every arc $a \in A(D)$ lies in a subdigraph $H_{a}$ of $D$, where $H_{a} \in \mathcal{H}$. For convenience, we also call a locally $\{H\}$ digraph $D$ as a locally $H$ digraph. A digraph $D$ is quasi-transitive if, for every triple of distinct vertices $x, y, z \in V(D)$, with $(x, y),(y, z) \in A(D)$, there is at least one arc between $x$ and $z$. A digraph $D$ is semicomplete if there is an arc between every pair of vertices in $D$. A digraph $D=(V, A)$ is semicomplete multipartite if there is a partition $V_{1}, V_{2}, \ldots, V_{c}$ of $V$ into independent sets so that every vertex in $V_{i}$ shares an arc with every vertex in $V_{j}$ for $1 \leq i<j \leq c$.

Motivated by the Chinese Postman Problem, Boesch, Suffel, and Tindell [30] in 1977 proposed the supereulerian problem, which seeks to characterize graphs that have spanning Eulerian subgraphs, and they [30] indicated that this problem would be very difficult. Pulleyblank [26] later in 1979 proved that determining whether a graph is supereulerian, even within planar 
graphs, is NP-complete. Since then, there have been lots of researches on this topic. Catlin [20] in 1992 presented the first survey on supereulerian graphs. Later Chen et al [21] gave an update in 1995, specifically on the reduction method associated with the supereulerian problem. A recent survey on supereulerian graphs is given in [24].

It is a natural to consider the supereulerian problem in digraphs. A digraph $D$ is eulerian if $D$ is connected and for any $v \in V(D), d_{D}^{+}(v)=d_{D}^{-}(v)$. A digraph $D$ is supereulerian if $D$ contains a spanning eulerian subdigraph. A digraph $D$ is a closed ditrail if it is eulerian. The main problems are to investigate the number of dicycles needed to cover a Hamiltonian oriented graph and to determine supereulerian digraphs.

\subsection{Previous Work On Supereulerian Digraphs}

Several efforts in supereulerian digraphs have been made. However, contrary to the case of undirected graphs, not much work has been done yet for supereulerian digraphs. The earlier studies were done by Gutin ( [31,32]). In [32], G.Gutin stated the following definitions:

Definition 1.2.1. Given a digraph $D$ and two positive integers $f(x), g(x)$ for every $x \in V(D), a$ subgraph $H$ of $D$ is called $a(g, f)$-factor if $g(x) \leq d_{H}^{+}(x)=d_{H}^{-}(x) \leq f(x)$ for every $x \in V(D)$. If $f(x)=g(x)=1$ for every $x$, then a connected $(g, f)$-factor is a hamiltonian cycle.

Definition 1.2.2. An extension of a digraph $D$ is a new digraph $H$ obtained from $D$ by replacing every vertex $x \in V(D)$ with a set of independent vertices $S_{x}$ such that, for every pair of distinct $x, y \in V(D)$, an arc $(u, v)$, where $u \in S_{x}, y \in S_{y}$, is in $H$ if and only if $(x, y)$ is in $D$. An extension of a locally in-semicomplete digraph is called an extended locally in - semicomplete digraph.

Then he proved the following theorem:

Theorem 1.2.3. (Gutin [32]) Let D be a semicomplete bipartite digraph or an extended locally in - semicomplete digraph. Then $D$ has a connected $(g, f)$-factor if and only if $D$ is strongly connected and contains a $(g, f)$-factor.

Using definition1.2.1 Gutin added that: Clearly, a digraph $H$ is supereulerian if and only if $H$ has a connected $\left(1, d^{*}\right)$-factor, where $d^{*}=\min \left\{d^{+}(x), d^{-}(x)\right\}$ for every $x \in V(H)$. As a result he presented the following corollary: 
Corollary 1.2.4. (Gutin [32]) Let D be a semicomplete bipartite digraph or an extended locally in - semicomplete digraph. $D$ is sup ereulerian if and only if $D$ is strongly connected and contains a $\left(1, d^{*}\right)$-factor.

A well known theorem of Chvátal and Erdös [22] states that if $|V(G)| \geq 3$ and if $\kappa(G) \geq$ $\alpha(G)$, then $G$ is hamiltonian. Thomassen [25] gave an infinite family of non hamiltonian (but supereulerian) digraphs such that $\kappa(D)=\alpha(D)=2$, showing that the Chvátal-Erdös Theorem does not extend to digraphs. This motivates Bang-Jensen and Thommassé in [17] to make the following conjecture.

Conjecture 1.2.5. Let $D$ be a digraph. If $\lambda(D) \geq \alpha(D)$, then $D$ is supereulerian.

Moreover in [17] Bang-Jensen and Alessandro Maddaloni analyzed a number of sufficient conditions for a digraph to be supereulerian starting with the following definition:

Definition 1.2.6. Given a digraph $D$, a dicycle factor, eulerian factor of $D$ is a collection of vertex-disjoint, arc-disjoint, respectively dicycles spanning $V(D)$.

Then as an effort towards conjecture 1.2.5 they proved the following theorem:

Theorem 1.2.7. (J. Bang-Jensen and A. Maddaloni [17]) Let $D$ be a digraph. If $\lambda(D) \geq \alpha(D)$ then $D$ has an eulerian factor.

In [17], Conjecture 1.2.5 has been verified in several families of digraphs. Gutin in [31] proved the following theorem:

Theorem 1.2.8. (Gutin [31]) An extended semicomplete digraph is hamiltonian if and only if it is strong and has a cycle factor.

Using a similar approach to Gutin's proof of Theorem 1.2.8, Bang-Jensen and Alessandro Maddaloni in [17] proved the following supereulerian version of it.

Theorem 1.2.9. (J. Bang-Jensen and A. Maddaloni [17]) Let D be a semicomplete multipartite digraph. $D$ is supereulerian if and only if it is strong and has an eulerian factor.

Combining Theorem 1.2.9 and Theorem 1.2.7, Bang-Jensen and Alessandro Maddaloni verify Conjecture 1.2.5 for semicomplete multipartite digraphs.

Theorem 1.2.10. (J. Bang-Jensen and A. Maddaloni [17]) Let D be a semicomplete multipartite digraph. If $\lambda(D) \geq \alpha(D)$, then $D$ is supereulerian. 
In addition, Bang-Jensen and Alessandro Maddaloni proved that:

Theorem 1.2.11. (J. Bang-Jensen and A. Maddaloni [17]) Let D be a quasi transitive digraph. If $\lambda(D) \geq \alpha(D)$, then $D$ is supereulerian.

Also, Bang-Jensen and Alessandro Maddaloni proved the following:

Theorem 1.2.12. (J. Bang-Jensen and A. Maddaloni [17]) A strong digraph such that $d(x)+$ $d(y) \geq 2 n-3$ for all pairs of non-adjacent vertices $x, y$ is supereulerian.

Corollary 1.2.13. (J. Bang-Jensen and A. Maddaloni [17]) A strong digraph such that $d^{+}(x)+$ $d^{-}(y) \geq n-1$ for all ordered pairs $(x, y)$ of non-adjacent vertices is supereulerian.

Finally, Bang-Jensen and Alessandro Maddaloni proved that the condition in congecture 1.2.5 is not necesary by the following example: consider a directed cycle on 4 vertices $C_{4}$ : it is eulerian, and hence supereulerian, but $\lambda\left(C_{4}\right)=1$ and $\alpha\left(C_{4}\right)=2$. Moreover, they provided infinite families of digraphs with $\lambda(D)=\alpha(D)-1$ that are not supereulerian. Hence, if true, Congecture 1.2.5 would be best possible.

In [34], Yanmei Hong, Hong-Jian Lai and Qinghai Liu after producing the following definition:

Definition 1.2.14. Let $D$ be a strong digraph and $U \subsetneq V(D)$. Then in $D[U]$, we can find some ditrails $P_{1}, \ldots, P_{t}$ such that $\cup_{i=1}^{t} V\left(P_{i}\right)=U$ and $A\left(P_{i}\right) \cap A\left(P_{j}\right)=\emptyset$ for any $i \neq j$. Let $\tau(U)$ be minimum value of such $t$. Then $c(G(D[U])) \leq \tau(U) \leq|U|$, where $c(G(D[U]))$ is the number of component of the underlying graph of $D[U]$. For any $A \subseteq V(D)-U$, denote $B:=V(D)-U-A$ and let

$$
\begin{aligned}
h(U, A): & =\min \left\{\left|\partial_{D}^{+}(A)\right|,\left|\partial_{D}^{-}(A)\right|\right\}+\min \left\{\left|(U, B)_{D}\right|,\left|(B, U)_{D}\right|\right\}-\tau(U), \text { and } \\
h(U): & =\min \{h(U, A): A \cap U=\emptyset\} .
\end{aligned}
$$

Yanmei Hong, Hong-Jian Lai and Qinghai Liu gave the following necessary condition for the existence of a spanning eulerian subdigraph:

Proposition 1.2.15. (Proposition (2.1) of Hong et al [34]) If D has a spanning eulerian subdigraph, then for any $U \subset V(D), h(U) \geq 0$.

Moreover, Hong et al gave the following sufficient degree condition for the existence of a spanning eulerian subdigraph: 
Theorem 1.2.16. (Hong et al [34]) Let D be a strong digraph of order $n$ and minimum outdegree $\delta^{+} \geq 4$ and minimum in-degree $\delta^{-} \geq 4$. If $\delta^{+}+\delta^{-}>n-4$, then $D$ has a spanning eulerian subdigraph.

\subsection{Main Results}

The main results in the dissertation are summarized as follows.

1. A dicycle cover of a digraph $D$ is a family $\mathcal{F}$ of dicycles of $D$ such that each arc of $D$ lies in at least one dicycle in $\mathcal{F}$. We investigate the problem of determining the upper bounds for the minimum number of dicycles which cover all arcs in a strong digraph. Best possible upper bounds of dicycle covers are obtained in a number of classes of digraphs, including strong tournaments, Hamiltonian oriented graphs, Hamiltonian oriented complete bipartite graphs, and families of possibly non-hamiltonian digraphs obtained from these digraphs via a sequence of 2 -sum operations.

2. Catlin in 1988 indicated that there exist graph families $\mathcal{F}$ such that if every edge $e$ in a graph $G$ lies in a subgraph $H_{e}$ of $G$ isomorphic to a member in $\mathcal{F}$, then $G$ is supereulerian. In particular, if every edge of a connected graph $G$ lies in a 3-cycle, then $G$ is supereulerian. The purpose of this research is to investigate how Catlin's theorem can be extended to digraphs. A strong digraph $D$ is supereulerian if $D$ contains a spanning eulerian subdigraph. We show that there exists an infinite family of non-supereulerian strong digraphs each arc of which lies in a directed 3-cycle. We also show that there exist digraph families $\mathcal{H}$ such that a strong digraph $D$ is supereulerian if every arc $a$ of $D$ lies in a subdigraph $H_{a}$ isomorphic to a member of $\mathcal{H}$. A digraph $D$ is symmetric if $(x, y) \in A(D)$ implies $(y, x) \in A(D)$; and is symmetrically connected if every pair of vertices of $D$ are joined by a symmetric dipath. A digraph $D$ is partially symmetric if the digraph obtained from $D$ by contracting all symmetrically connected components is symmetrically connected. It is known that a partially symmetric digraph may not be symmetrically connected. We show that symmetrically connected digraphs and partially symmetric digraphs are such families. Sharpness of these results are discussed.

3. The 2-sum of two digraphs $D_{1}$ and $D_{2}$, denoted $D_{1} \oplus_{2} D_{2}$, is the digraph obtained from the disjoint union of $D_{1}$ and $D_{2}$ by identifying an arc in $D_{1}$ with an arc in $D_{2}$. A digraph $D$ is supereulerian if $D$ contains a spanning eulerian subdigraph. It has been noted that the 2-sum of two supereulerian (or even hamiltonian) digraphs may not be supereulerian. 
We obtain several sufficient conditions on $D_{1}$ and $D_{2}$ for $D_{1} \oplus_{2} D_{2}$ to be supereulerian. In particular, we show that if $D_{1}$ and $D_{2}$ are symmetrically connected or partially symmetric, then $D_{1} \oplus_{2} D_{2}$ is supereulerian. 


\section{Chapter 2}

\section{Dicycle cover of Hamiltonian oriented graphs}

\subsection{The problem}

We consider finite loopless graphs and digraphs, and undefined notations and terms will follow [1] for graphs and [2] for digraphs. In particular, a cycle is a 2-regular connected nontrivial graph. A cycle cover of a graph $G$ is a collection $\mathcal{C}$ of cycles of $G$ such that $E(G)=\bigcup_{C \in \mathcal{C}} E(C)$. Bondy [4] conjectured that if $G$ is a 2-connected simple graph with $n \geq 3$ vertices, then $G$ has a cycle cover $\mathcal{C}$ with $|\mathcal{C}| \leq \frac{2 n-3}{3}$. Bondy [4] showed that this conjecture, if proved, would be best possible. Y. X. Luo and R. S. Chen [14] proved that this conjecture holds for 2-connected simple cubic graphs. It has been shown that for plane triangulations, serial-parallel graphs or planar graphs in general, one can have a better bound for the number of cycles used in a cover ( [9-12]). Barnette [3] proved that if $G$ is a 3-connected simple planar graph of order $n$, then

the edges of $G$ can be covered by at most $\frac{n+1}{2}$ cycles. Fan [6] settled this conjecture by showing that it holds for all simple 2 -connected graphs. The best possible number of cycles needed to cover cubic graphs has been obtained in [13] and [15].

A directed path in a digraph $D$ from a vertex $u$ to a vertex $v$ is called a $(u, v)$-dipath. To emphasize the distinction between graphs and digraphs, a directed cycle or path in a digraph is often referred as a dicycle or dipath. It is natural to consider the number of dicycles needed to cover a digraph. Following [2], for a digraph $D, V(D)$ and $A(D)$ denote the vertex set and arc set of $D$, respectively. If $A^{\prime} \subseteq A(D)$, then $D\left[A^{\prime}\right]$ is the subdigraph induced by $A^{\prime}$. Let $K_{n}^{*}$ 
denote the complete digraph on $n$ vertices. Any simple digraph $D$ on $n$ vertices can be viewed as a subdigraph of $K_{n}^{*}$. If $W$ is an arc subset of $A\left(K_{n}^{*}\right)$, then $D+W$ denotes the digraph $K_{n}^{*}[A(D) \cup W]$.

A digraph $D$ is strong if for any distinct $u, v \in V(D), D$ has a $(u, v)$-dipath. As in $[2], \lambda(D)$ denotes the arc-strong-connectivity of $D$. Thus a digraph $D$ is strong if and only if $\lambda(D) \geq 1$. We use $(u, v)$ denotes an arc with tail $u$ and head $v$. For $X, Y \subseteq V(D)$, we define

$$
(X, Y)_{D}=\{(x, y) \in A(D): x \in X \text { and } y \in Y\} ; \text { and } \partial_{D}^{+}(X)=(X, V(D)-X)_{D}
$$

Let

$$
d_{D}^{+}(X)=\left|\partial_{D}^{+}(X)\right|, \text { and } d_{D}^{-}(X)=\left|\partial_{D}^{-}(X)\right|
$$

When $X=\{v\}$, we write $d_{D}^{+}(v)=\left|\partial_{D}^{+}\{v\}\right|$ and $d_{D}^{-}(v)=\left|\partial_{D}^{-}\{v\}\right|$. Let $N_{D}^{+}(v)=\{u \in V(D)-v$ : $(v, u) \in A(D)\}$ and $N_{D}^{-}(v)=\{u \in V(D)-v:(u, v) \in A(D)\}$ denote the out-neighbourhood and in-neighbourhood of $v$ in $D$, respectively. We call the vertices in $N_{D}^{+}(v), N_{D}^{-}(v)$ the out-neighbours, in-neighbours of $v$. Thus for a digraph $D, \lambda(D) \geq 1$ if and only if for any proper non empty subset $\emptyset \neq X \subset V(D),\left|\partial_{D}^{+}(X)\right| \geq 1$.

A dicycle cover of a digraph $D$ is a collection $\mathcal{C}$ of dicycles of $D$ such that $\bigcup_{C \in \mathcal{C}} A(C)=$ $A(D)$. If $D$ is obtained from a simple undirected graph $G$ by assigning an orientation to the edges of $G$, then $D$ is an oriented graph. The main purpose is to investigate the number of dicycles needed to cover a Hamiltonian oriented graph. We prove the following.

Theorem 2.1.1. Let $D$ be an oriented graph on $n$ vertices and $m$ arcs. If $D$ has a Hamiltonian dicycle, then $D$ has a dicycle cover $\mathcal{C}$ with $|\mathcal{C}| \leq m-n+1$. This bound is best possible.

In the next section, we will first show that every Hamiltonian oriented graph with $n$ vertices and $m$ arcs can be covered by at most $m-n+1$ dicycles. Then we show that for every Hamiltonian graph $G$ with $n$ vertices and $m$ edges, there exists an orientation $D=D(G)$ of $G$ such that any dicycle cover of $D$ must have at least $m-n+1$ dicycles.

\subsection{Proof of the Main Result}

In this section, all graphs are assumed to be simple. We start with an observation, stated as a Lemma below. A digraph $D$ is weakly connected if the underlying graph of $D$ is connected. 
Lemma 2.2.1. A weakly connected digraph $D$ has a dicycle cover if and only if $\lambda(D) \geq 1$.

Proof. Suppose $D$ has a dicycle cover $\mathcal{C}$. If $D$ is not strong, then there exists a proper nonempty subset $\emptyset \neq X \subset V(D)$ such that $\left|\partial_{D}^{+}(X)\right|=0$. Since $D$ is weakly connected, $D$ contains an arc $(u, v) \in(V(D)-X), X)_{D}$. Since $\mathcal{C}$ is a dicycle cover of $D$, there exists a dicycle $C \in \mathcal{C}$ with $(u, v) \in A(C)$. Since $(u, v) \in(V(D)-X), X)_{D}$, we conclude that $\left.\emptyset \neq A(C) \cap(X, V(D)-X)\right)_{D} \subseteq$ $\partial_{D}^{+}(X)$, contrary to the assumption $\left|\partial_{D}^{+}(X)\right|=0$. This proves that $D$ must be strong.

Conversely, assume that $D$ is strong. For any arc $a=(u, v) \in A(D)$, since $D$ is strong, there must be a directed $(v, u)$-path $P$ in $D$. It follows that $C_{a}=P+a$ is a dicycle of $D$ containing $a$, and so $\left\{C_{a}: a \in A(D)\right\}$ is a dicycle cover of $D$.

Let $C$ be a dicycle and let $a=(u, v)$ be an arc not in $A(C)$ but with $u, v \in V(C)$. Then $C+a$ contains a unique dicycle $C_{a}$ containing $a$. In the following, we call $C_{a}$ the fundamental dicycle of $a$ with respect to $C$.

Lemma 2.2.2. Let $D$ be an oriented graph on $n$ vertices and $m$ arcs. If $D$ has a Hamiltonian dicycle, then $D$ has a dicycle cover $\mathcal{C}$ with $|\mathcal{C}| \leq m-n+1$.

Proof. Let $C_{0}$ denote the directed Hamiltonian cycle of $D$. For each $a \in A(D)-A(C)$, let $C_{a}$ denote the fundamental dicycle of $a$ with respect to $C$. Then $\mathcal{C}=\left\{C_{0}\right\} \cup\left\{C_{a}: a \in A(D)-A(C)\right\}$ is a dicycle cover of $D$ with $|\mathcal{C}| \leq m-n+1$.

In the following, we present some concepts and tools needed to construct such an example of Hamiltonian oriented graphs to show the inequality of Theorem 2.1.1 is best possible.

Definition 2.2.3. Let $D$ be a directed graph. A topological order of a digraph $D$ is an order of vertices of $V(D)=\left\{v_{1}, v_{2}, \ldots, v_{n}\right\}$ such that for each edge $\left(v_{i}, v_{j}\right) \in E(D)$ we have $i<j$.

Proposition 2.2.4. A directed graph $D$ has a topological order if and only if $D$ is acyclic.

The undirected graph say $G$ will be acyclic digraph, by assigning an orientation to the edges of graph $G$ based on Proposition 2.2.4, as the following:

\section{- A cyclic Algorithm:}


- Input: A undirected simple graph G.

- Output: A cyclic orientation of simple graph G.

* $\operatorname{Step(1):~Order~the~vertices~of~} V(G)=\left\{v_{1}, v_{2}, \ldots, v_{n}\right\}$ in the clockwise direction.

* Step(2): Oriented all edges of $E\left(v_{1}\right)$ as out edges.

* $\operatorname{Step(3):~Oriented~all~edges~of~} E\left(v_{2}\right)$ as out edges.

* $\operatorname{Step(4):~After~oriented~} E\left(v_{1}\right) \cup E\left(v_{2}\right) \cup \ldots \cup E\left(v_{k}\right)$ oriented the edges of $E\left(v_{k+1}\right)-$ $\left(\bigcup_{i=1}^{k} E\left(v_{i}\right)\right)$ as out edges.

Let $G$ be a Hamiltonian graph, We need to construct an orientation $D$ for a graph $G$ so that $A(D(G))-A(C)$ be a cyclic digraph. The structured way to construct such an orientation $D$ by using the following approaches :

- Approach (1): Oriented a Hamilton cycle $C$.

- Approach (2): Oriented all edges of $E(G)-E(C)$ by using acyclic algorithm.

According to the approach (1), there are two ways to assign a directions to the edges of a Hamilton cycle $C$. Let define such of these directions as as the following:

- $D(C):=$ Oriented the edges in the a Hamilton cycle $C$ in the anticlockwise direction.

- $D^{\prime}(C):=$ Oriented the edges in the a Hamilton cycle $C$ in the clockwise direction

In the following table show the difference number of dicycle cover for the Hamilton oriented graph $D(G)$ according to the orientation of a Hamilton cycle $C$. 


\begin{tabular}{|r|c|c|l|}
\hline \hline $\mathbf{n}$ & $d c c(D(G))$ & $D^{\prime}(C)$ & $D(C)$ \\
\hline 5 & 6 & 5 & 7 \\
6 & 10 & 8 & 11 \\
7 & 15 & 11 & 15 \\
8 & 21 & 15 & 21 \\
9 & 28 & 19 & 28 \\
10 & 36 & 25 & 38 \\
11 & 45 & 29 & 45 \\
12 & 55 & 35 & 55 \\
13 & 66 & 42 & 68 \\
14 & 78 & 48 & 78 \\
15 & 91 & 55 & 91 \\
16 & 105 & 63 & 105 \\
\hline
\end{tabular}

To prove Theorem 2.1.1 is best possible, we need to construct, for each integer $n \geq 4$, a Hamiltonian oriented graph on $n$ vertices and $m \operatorname{arcs} D$ such that any dicycle cover $\mathcal{C}$ of $D$ must have at least $m-n+1$ dicycles in $\mathcal{C}$.

We present a summary of our structure as Definition below. Let $G$ be a Hamiltonian simple graph. We present a construction of such an orientation $D=D(G)$. Since $G$ is Hamiltonian, we may assume that $V(G)=\left\{v_{1}, v_{2}, \ldots, v_{n}\right\}$ and $C=v_{1} v_{2}, \ldots, v_{n} v_{1}$ is a Hamiltonian cycle of $G$.

Definition 2.2.5. Define an orientation $D=D(G)$ as follows.

(i) Orient the edges in the Hamiltonian cycle $C=v_{1} v_{2}, \ldots, v_{n} v_{1}$ as follows.

$$
\left(v_{i+1}, v_{i}\right) \in A(D), i=1,2, \ldots, n-1,\left(v_{1}, v_{n}\right) \in A(D) .
$$

(ii) For each $i=2,3, \ldots, n-2$, and for each $j=i+2, i+3, \ldots n$, assign directions to edges of $G$ not in $E(C)$ as follows.

$$
\left(v_{i}, v_{j}\right) \in A(D) \text {, if } v_{i} v_{j} \in E(G)-E(C) \text { and } i+1<j \leq n \text {. }
$$

and

$$
\left(v_{1}, v_{j}\right) \in A(D), \text { if } v_{1} v_{j} \in E(G)-E(C) \text { and } i+1<j \leq n-1
$$




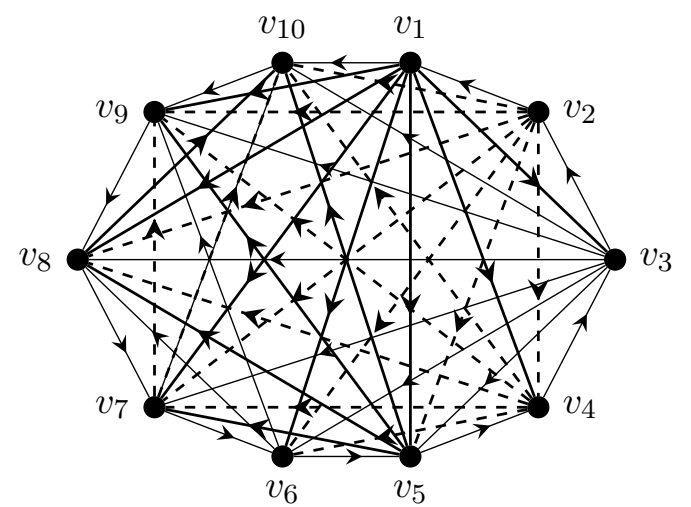

Figure 1: An orientation of a complete graph $D\left(K_{10}\right)$.

We make the following observations stated in the Lemma below.

Lemma 2.2.6. Each of the following holds for the digraph $D$.

(i) The dicycle $C_{0}=v_{1} v_{n} v_{n-1} \ldots v_{3} v_{2} v_{1}$ is a Hamiltonian dicycle of $D$.

(ii) The digraph $D-A\left(C_{0}\right)$ is acyclic.

(iii) $N_{D}^{+}\left(v_{n}\right)=\left\{v_{n-1}\right\} ; N_{D}^{-}\left(v_{1}\right)=\left\{v_{2}\right\} ; N_{D}^{-}\left(v_{2}\right)=\left\{v_{3}\right\}$.

(iv) The dicycle $C_{0}$ is the only dicycle of $D$ containing the arc $\left(v_{1}, v_{n}\right)$.

(v) The dicycle $C_{0}$ is the unique Hamiltonian dicycle of $D$.

(vi) If $C^{\prime \prime}$ is a dicycle of $D$, then $C^{\prime \prime}$ contains at most one arc in $A(D)-A\left(C_{0}\right)$.

Proof. (i). Follows immediately from Definition 2.2.5 (i).

(ii). By Definition 2.2.5, the labels of the vertices $V(D)=\left\{v_{1}, v_{2}, \ldots, v_{n}\right\}$ satisfies $\left(v_{i}, v_{j}\right) \in$ $A(D)-A\left(C_{0}\right)$ only if $i<j$. It follows (for example, Section 2.1 of [2]) that $D-A\left(C_{0}\right)$ is acyclic, and so (ii) holds.

(iii). This follows immediately from Definition 2.2.5.

(iv). Let $C^{\prime}$ be a dicycle of $D$ with $\left(v_{1}, v_{n}\right) \in A\left(C^{\prime}\right)$. Since $\left(v_{1}, v_{n}\right) \in A\left(C^{\prime}\right) \cap A\left(C_{0}\right)$, we choose the largest label $i \leq n$, such that $\left(v_{1}, v_{n}\right),\left(v_{n}, v_{n-1}\right), \ldots,\left(v_{i+1}, v_{i}\right) \in A\left(C^{\prime}\right) \cap A\left(C_{0}\right)$. Since $C^{\prime} \neq C_{0}$, we have $i \geq 3$. Since $C^{\prime}$ is a dicycle, there must be a vertex $v_{j} \in V(D) \operatorname{such}$ that $\left(v_{i}, v_{j}\right) \in A\left(C^{\prime}\right)$. By the choice of $i$, we must have $\left(v_{i}, v_{j}\right) \notin A\left(C_{0}\right)$, and so $\left(v_{i}, v_{j}\right) \in A(D)-A\left(C_{0}\right)$. By Definition 2.2 .5 (ii), we have $i+2 \leq j \leq n$, contrary to the fact that $C^{\prime}$ is a dicycle of $D$ containing $\left(v_{1}, v_{n}\right)$. This proves (iv).

(v). Let $C^{\prime}$ be a Hamiltonian dicycle of $D$. Since $V\left(C^{\prime}\right)=V(D)$, we have $v_{n} \in V\left(C^{\prime}\right)$. We claim that $\left(v_{1}, v_{n}\right) \in A\left(C^{\prime}\right)$. If $\left(v_{1}, v_{n}\right) \notin A\left(C^{\prime}\right)$, then there exists a $v_{i} \in V(C)\left(i \in\left\{v_{2}, v_{3}, \ldots, v_{n-1}\right\}\right)$ such that $\left(v_{i}, v_{n}\right) \in A\left(C^{\prime}\right)$. Hence, $\left(v_{i}, v_{n}\right),\left(v_{n}, v_{n-1}\right), \ldots,\left(v_{i+2}, v_{i+1}\right) \in A\left(C^{\prime}\right)$. By Definition 2.2 .5 (i) and (ii), $N^{+}\left(v_{i+1}\right) \subset\left\{v_{i+2}, v_{i+3}, \ldots, v_{n}\right\}$, contrary to the fact that $C^{\prime}$ is a Hamiltonian 
dicycle of $D$. Thus, $\left(v_{1}, v_{n}\right) \in A\left(C^{\prime}\right)$. It follows by Lemma 2.2.6 (iv) that we must have $C^{\prime}=C_{0}$,

(vi). By contradiction, we assume that $D$ has a dicycle $C^{\prime \prime}$ which contains two $\operatorname{arcs} a_{1}, a_{2} \in$ $A(D)-A\left(C_{0}\right)$. Since $V(D)=\left\{v_{1}, v_{2}, \ldots, v_{n}\right\}$, we assume that $a_{1}=\left(v_{i}, v_{i^{\prime}}\right)$ and $a_{2}=\left(v_{j}, v_{j^{\prime}}\right)$. Without lost of generality and by Lemma 2.2.5, we further assume that $1 \leq i<j<n$.

Let $i \geq t \geq 1$ be the smallest integer such that $v_{t} \in V\left(C^{\prime \prime}\right)$. Since $C^{\prime \prime}$ is a dicycle of $D$, there must be a $v_{s} \in V\left(C^{\prime \prime}\right)$ such that $\left(v_{s}, v_{t}\right) \in A\left(C^{\prime \prime}\right)$. By Definition 2.2.5, either $\left(v_{s}, v_{t}\right) \in A\left(C_{0}\right)$ and $s=t+1<j$ or $\left(v_{s}, v_{t}\right) \in A(D)-A\left(C_{0}\right)$ and $1<s+1<t$. By the choice of $t$, we can only have $s=t+1$ and $\left(v_{t+1}, v_{t}\right) \in A\left(C^{\prime \prime}\right) \cap A\left(C_{0}\right)$. Choose the largest integer $h$ with $t+1 \leq h<j$ such that $\left(v_{t+1}, v_{t}\right),\left(v_{t+2}, v_{t+1}\right), \ldots,\left(v_{h}, v_{h-1}\right) \in A\left(C^{\prime \prime}\right) \cap A\left(C_{0}\right)$. Since $C^{\prime \prime}$ is a dicycle, there must be a $v_{k}$ with $1 \leq k \leq n$ such that $\left(v_{k}, v_{h}\right) \in A\left(C^{\prime \prime}\right)$. By the maximality of $h$ and by Definition2.2.5 (i), we conclude that $\left(v_{k}, v_{h}\right) \notin A\left(C_{0}\right)$. By Definition2.2.5 (ii), $1 \leq k \leq h-2$. By the minimality of $t$, we must have $t \leq k \leq h-2$. It follows by $j>h$ that $C^{\prime \prime}$ cannot contain $a_{2}=\left(v_{j}, v_{j^{\prime}}\right)$, contrary to the assumption. This contradiction justifies (vi).

To complete the proof of Theorem 2.1.1, we present the next Lemma.

Lemma 2.2.7. Let $G$ be a Hamiltonian simple graph. There exists an orientation $D=D(G)$ such that every dicycle cover of $D$ must have at least $m-n+1$ dicycles.

Proof. Let $G$ be a Hamiltonian graph and let $D=D(G)$ be the orientation of $G$ given in Definition 2.2.5. For notational convenience, we adopt the notations in Definition 2.2 .5 and denote $V(D)=\left\{v_{1}, v_{2}, \ldots, v_{n}\right\}$. Thus by Lemma 2.2.6 (v), $C_{0}=v_{1} v_{n} v_{n-1} \ldots v_{2} v_{1}$ is the unique Hamiltonian dicycle of $D$.

Let $\mathcal{C}$ be a dicycle cover of $D$. By Lemma 2.2.6 (iv), we must have $C_{0} \in \mathcal{C}$. For each arc $a \in A(D)-A\left(C_{0}\right)$, since $\mathcal{C}$ is a dicycle cover of $D$, there must be a dicycle $C(a) \in \mathcal{C}$ such that $a \in A(C(a))$. By Lemma 2.2.6 (vi), $A(C(a)) \cap A(D)-A\left(C_{0}\right)=\{a\}$. It follows that if $a, a^{\prime} \in A(D)-A\left(C_{0}\right)$, then $a \neq a^{\prime}$ implies $C(a) \neq C\left(a^{\prime}\right)$ in $\mathcal{C}$. Thus we have $\{C(a) \mid a \in$ $\left.A(D)-A\left(C_{0}\right)\right\} \subseteq \mathcal{C}$. Hence

$$
|\mathcal{C}| \geq\left|\left\{C(a): a \in A(D)-A\left(C_{0}\right)\right\} \cup\left\{C_{0}\right\}\right|=m-n+1 .
$$

This proves the Lemma.

By Lemmas 2.2.2 and 2.2.7, Theorem 2.1.1 follows. 
We are about to show that Theorem 2.1.1 can be applied to obtain dicycle cover bounds for certain families of oriented graphs.

\subsubsection{Tournament}

Definition 2.2.8. A tournament is an orientation of a complete graph $K_{n}$. An alternate definition for a tournament is for any two distinct vertices $u, v \in V\left(K_{n}\right)$ one of these arcs belong to $E\left(K_{n}\right)$ either $(u, v) \in E\left(K_{n}\right)$ or $(v, u) \in E\left(K_{n}\right)$ not all.

Let $T_{n}$ denote a tournament of order $n$. Then $T_{n}$ is an oriented graph.

Lemma 2.2.9. If $T_{n}$ is $k$-strong, then every length $(k-1)$ dipath can be extended to a Hamiltonian dicycle.

Proof. For $k=1$, Camion $[5,7]$ proved that every strong tournament is Hamiltonian.

For $k=2$, we have a counterexample $T_{6}$ show that $T_{6}$ is a 2 - strong tournament, but there exist an arc $a \in A\left(T_{6}\right)$ lies between the two vertices $v_{4}$ and $v_{6}$, can not be extended to a Hamilton dicycle. See Figure 2 .
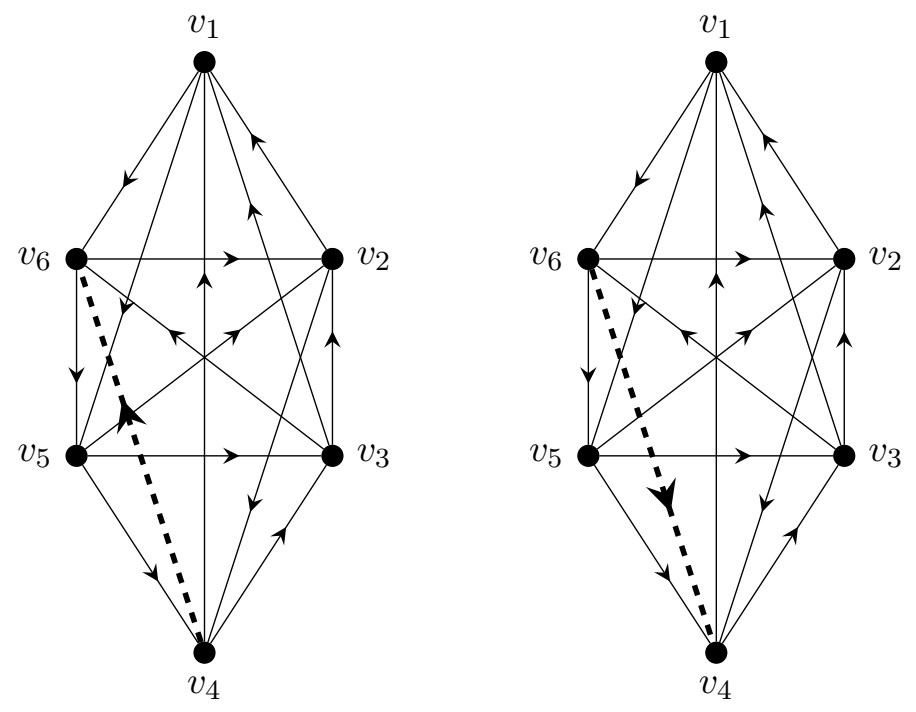

Figure 2: An arc $a \in A\left(T_{6}\right)$ can not extend to a Hamiltonian dicycle.

For $k=3$, we have a counterexample $T_{7}$ show that $T_{7}$ is a 3 - strong tournament, but there exist a dipath $P=\{(x, y),(y, z)\}$ with length 2 , can not be extended to a Hamilton dicycle. See Figure 3. 


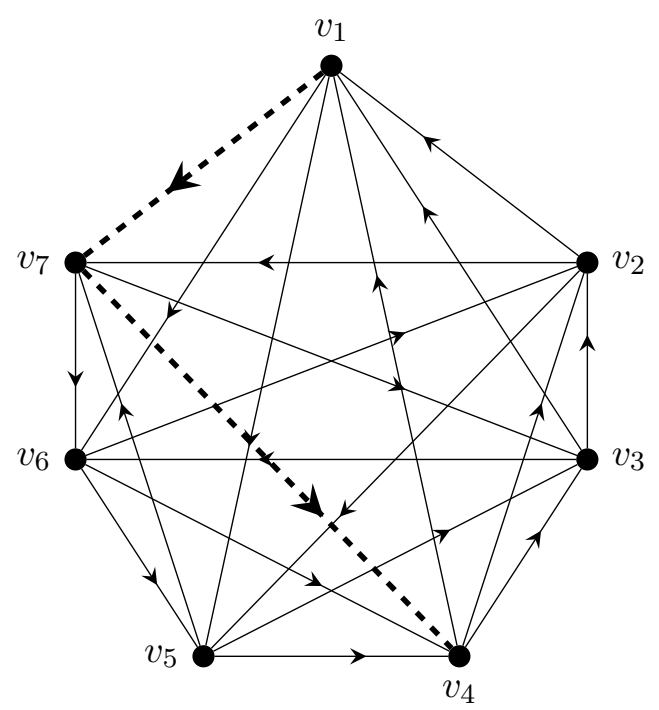

Figure 3: A dipath $P=\left\{\left(v_{1}, v_{7}\right),\left(v_{7}, v_{4}\right\}\right.$ in $T_{7}$ can not extend to a Hamiltonian dicycle.

Due to Camion Theorem, hence the corollary below follows from Theorem 2.1.1.

Corollary 2.2.10. Every strong tournament on $n$ vertices has a dicycle cover $\mathcal{C}$ with $|\mathcal{C}| \leq$ $\frac{n(n-1)}{2}-n+1$. This bound is best possible.

\subsubsection{A Complete Bipartite}

Definition 2.2.11. A bipartite graph $G$ with vertex bipartition $(A, B)$ is balanced if $|A|=|B|$.

Theorem 2.2.12. A bipartite graph $G$ with vertex bipartition $(A, B)$, has a Hamiltonian cycle if and only if $G$ is balanced.

Let $K_{m, n}$ be a complete bipartite graph with vertex bipartition $(A, B)$ and $|A|=m,|B|=n$ , then $K_{m, n}$ has Hamiltonian cycle if and only if $m=n \geq 2$, that is, $K_{m, n}$ is balanced. Let $K_{n, n}$ denote a balanced complete bipartite graph.

Corollary 2.2.13. Every Hamiltonian orientation of balanced complete bipartite graph $K_{n, n}$ has a dicycle cover $\mathcal{C}$ with $|\mathcal{C}| \leq(n-1)^{2}$. This bound is best possible.

Lemma 2.2.14. Let $K_{n, n}$ be a Hamiltonian orientation of balanced bipartite on $2 n$, then $D$ has a dicycle cover $\mathcal{C}$ with $|\mathcal{C}| \leq(n-1)^{2}$. 
Proof. Since a Hamiltonian orientation of balanced bipartite have $m=n^{2}$ and so by following Lemma 2.2.2 we have $|\mathcal{C}| \leq m-n+1 \leq n^{2}-2 n+1=(n-1)^{2}$.

To prove Corollary 2.2.13 is best possible, we have to construct, for $n \geq 3$ a Hamiltonian oriented balanced bipartite graph on $2 n$ such that any dicycle cover $\mathcal{C}$ of $K_{n, n}$ must have at least $(n-1)^{2}$ dicycle cover in $\mathcal{C}$.

The structured way to construct an orientation for a Hamiltonian oriented balanced bipartite graph on $2 n$ to be acyclic digraph by using the following steps :

- Step (1): Oriented a Hamilton cycle $C$.

- Step (2): Oriented all edges of $E\left(K_{n, n}\right)-E(C)$ by using acyclic algorithm.

According to the step (1), in the following table show the difference number of dicycle cover for the Hamiltonian oriented balanced bipartite graph $K_{n, n}$ according to the orientation of a Hamilton cycle $C$.

\begin{tabular}{|c|c|c|l|}
\hline \hline $\mathbf{n}$ & $d c c\left(K_{n, n}\right)$ & $D^{\prime}(C)$ & $D(C)$ \\
\hline 4 & 9 & 7 & 9 \\
5 & 16 & 12 & 16 \\
6 & 25 & 17 & 25 \\
7 & 36 & 24 & 36 \\
8 & 49 & 31 & 49 \\
9 & 64 & 40 & 64 \\
\hline
\end{tabular}

Now we can summarizing these steps into the following Definition. Let $k_{n, n}$ be a Hamiltonian balanced bipartite graph. We construct an orientation $D=D\left(k_{n, n}\right)$. Since $k_{n, n}$ is Hamiltonian balanced bipartite, we may assume that $A=\left\{u_{1}, u_{2}, \cdots, u_{n}\right\}, B=\left\{v_{1}, v_{2}, \cdots, v_{n}\right\}$ and $C=\left\{u_{1} v_{1} u_{2} v_{2} \cdots u_{n} v_{n} u_{1}\right\}$ is a Hamiltonian cycle of $k_{n, n}$.

Definition 2.2.15. We define an orientation $D=D\left(k_{n, n}\right)$ as following

(i) Orient the edges in the Hamiltonian dicycle $C=\left\{u_{1} v_{1} u_{2} v_{2} \cdots u_{n} v_{n} u_{1}\right\}$ as follows:

$$
\begin{gathered}
\left(v_{i}, u_{i}\right) \in A(D) \text { for } i=1,2, \cdots n . \\
\left(u_{i+1}, v_{i}\right) \in A(D) \text { for } i=1,2, \cdots n-1 . \\
\text { and }
\end{gathered}
$$




$$
\left(u_{1}, v_{n}\right) \in A(D)
$$

(ii) We orient to edges of $k_{n, n}$ not in $E(C)$ as follows:

$$
\begin{gathered}
\left(u_{j}, v_{i+j}\right) \in A(D) \text { for } i=1,2, \cdots n-j ; \text { and } j=1,2, \cdots n-1 . \\
\text { and } \\
\left(v_{l}, u_{i+(l+1)}\right) \in A(D) \text { for } i=1,2, \cdots n-(l+1) ; \text { and } l=1,2, \cdots n-2 .
\end{gathered}
$$

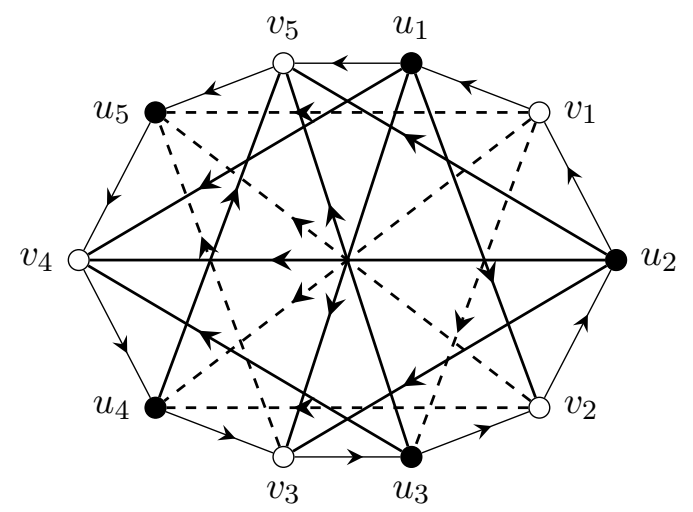

Figure 4: An orientation of a complete bipartite $D\left(K_{5,5}\right)$.

Remark 2.2.16. Since the orientation $D=D\left(k_{n, n}\right)$ of a Hamiltonian of balanced bipartite graph is similar to the orientation of Definition 2.2.5, then lemma 2.2.6 is still hold for $D=D\left(k_{n, n}\right)$.

To complete proof of Corollary 2.2.13, we present the following lemma. Thus Corollary 2.2.13 follows from lemmas 2.2.14 and 2.2.17.

Lemma 2.2.17. Let $k_{n, n}$ be a Hamiltonian of balanced bipartite graph with $n \geq 2$. There exist an orientation $D=D\left(k_{n, n}\right)$ such that every dicycle cover of $D$ must have at least $(n-1)^{2}$ dicycles.

Proof. Let $k_{n, n}$ be a Hamiltonian of balanced bipartite graph and let $D=D\left(k_{n, n}\right)$ be the orientation of $k_{n, n}$ given in Definition 2.2.15. By following Lemma 2.2.7, a Hamiltonian orientation of balanced bipartite have $m=n^{2}$ and hence $|\mathcal{C}| \geq m-n+1 \geq n^{2}-2 n+1=(n-1)^{2}$. 


\subsection{Dicycle Covers of 2-Sums of Digraphs}

In this section, we shall show that Theorem 2.1.1 can also be applied to certain non-hamiltonian digraphs which can be built via 2 -sums. We start with 2 -sums of digraphs.

Definition 2.3.1. Let $D_{n_{1}}=\left(V\left(D_{n_{1}}\right), A\left(D_{n_{1}}\right)\right)$ and $D_{n_{2}}=\left(V\left(D_{n_{2}}\right), A\left(D_{n_{2}}\right)\right)$ be two disjoint digraphs, $a_{1}=\left(v_{12}, v_{11}\right) \in A\left(D_{n_{1}}\right)$ and $a_{2}=\left(v_{22}, v_{21}\right) \in A\left(D_{n_{2}}\right)$. The 2-sum $D_{n_{1}} \oplus_{2} D_{n_{2}}$ of $D_{n_{1}}$ and $D_{n_{2}}$ is obtained from the union of $D_{n_{1}}$ and $D_{n_{2}}$ by identifying the arcs $a_{1}$ and $a_{2}$, that is, $v_{11}=v_{21}$ and $v_{12}=v_{22}$.

Definition 2.3.2. Let $D_{n_{1}}, D_{n_{2}}, \ldots, D_{n_{s}}$ be $s$ disjoint digraphs with $n_{1}, n_{2}, \ldots, n_{s}$ vertices, respectively. Let $D_{n_{1}} \oplus_{2} D_{n_{2}} \oplus_{2} \ldots \oplus_{2} D_{n_{s}}$ denote a sequence of 2-sum of $D_{n_{1}}, D_{n_{2}}, \ldots, D_{n_{s}}$, that is, $\left(\left(\left(D_{n_{1}} \oplus_{2} D_{n_{2}}\right) \oplus_{2} D_{n_{3}}\right) \oplus_{2} \ldots\right) \oplus_{2} D_{n_{s}}$.

Theorem 2.3.3. Let $D_{n_{1}}, D_{n_{2}}, \ldots, D_{n_{s}}$ be s disjoint Hamiltonian oriented graphs on $n_{1}, n_{2}, \ldots, n_{s}$ vertices and $m_{1}, m_{2}, \ldots, m_{s}$ arcs, respectively, and let $D=D_{n_{1}} \oplus_{2} D_{n_{2}} \oplus_{2} \ldots \oplus_{2} D_{n_{s}}$. Then $D$ has a dicycle cover $\mathcal{C}$ with $|\mathcal{C}| \leq|A(D)|-|V(D)|+1$. This bound is best possible.

Proof. By Theorem 2.1.1, $D_{n_{i}}(i=1,2, \ldots s)$ has a dicycle cover $\mathcal{C}_{i}$ with $\left|\mathcal{C}_{i}\right| \leq m_{i}-n_{i}+1$. Let $\mathcal{C}=\bigcup_{i=1}^{s} \mathcal{C}_{i}$. Then $|\mathcal{C}| \leq\left(m_{1}-n_{1}+1\right)+\left(m_{2}-n_{2}+1\right)+\ldots+\left(m_{s}-n_{s}+1\right)=\left(m_{1}+m_{2}+\ldots+\right.$ $\left.m_{s}\right)-\left(n_{1}+n_{2}+\ldots+n_{s}\right)+s=\left(m_{1}+m_{2}+\ldots+m_{s}-(s-1)\right)-\left(n_{1}+n_{2}+\ldots+n_{s}-2(s-1)\right)+1=$ $|A(D)|-|V(D)|+1$. By Definition 2.3.2, $\mathcal{C}$ is a dicycle cover of $D$. Thus, $D$ has a dicycle cover $\mathcal{C}$ with $|\mathcal{C}| \leq|A(D)|-|V(D)|+1$.

Let $G_{n_{i}}$ be $s$ disjoint Hamiltonian simple graphs for $i \in\{1,2, \ldots, s\}$. We may assume that $V\left(G_{n_{i}}\right)=\left\{v_{i 1}, v_{i 2}, \ldots, v_{i n_{i}}\right\}$ and $C_{i}=v_{i 1} v_{i 2} \ldots v_{i n_{i}} v_{i 1}$ is a Hamiltonian cycle of $G_{n_{i}}$, and let

$$
D_{n_{i}}=D\left(G_{n_{i}}\right) \text { be the orientation of } G_{n_{i}} \text { given in Definition 2.2.5. }
$$

For notational convenience, we adopt the notations in Definition 2.2.5 and denote $V\left(D_{n_{i}}\right)=$ $\left\{v_{i 1}, v_{i 2}, \ldots, v_{i n_{i}}\right\}$. Thus by Lemma 2.2.6 (v), $C_{i_{0}}=v_{i 1} v_{i n_{i}} \ldots v_{i 2} v_{i 1}$ is the unique Hamiltonian dicycle of $D_{n_{i}}$. Let $a_{i}=\left(v_{i 2}, v_{i 1}\right)$ is an arc of $D_{n_{i}}$. We construct the 2-sum digraph $D_{n_{1}} \oplus_{2}$ $D_{n_{2}} \oplus_{2} \ldots \oplus_{2} D_{n_{s}}$ from the union of $D_{n_{1}}, D_{n_{2}}, \ldots, D_{n_{s}}$ by identifying the $\operatorname{arcs} a_{1}, a_{2}, \ldots, a_{s}$ such that $v_{11}=v_{21}=\ldots=v_{s 1}, v_{12}=v_{22}=\ldots=v_{s 2}$. We assume that $v_{1}:=v_{11}=v_{21}=\ldots=v_{s 1}$, $v_{2}:=v_{12}=v_{22}=\ldots=v_{s 2}$. (The case when $s=2$ is depicted in Figure 5 ). 


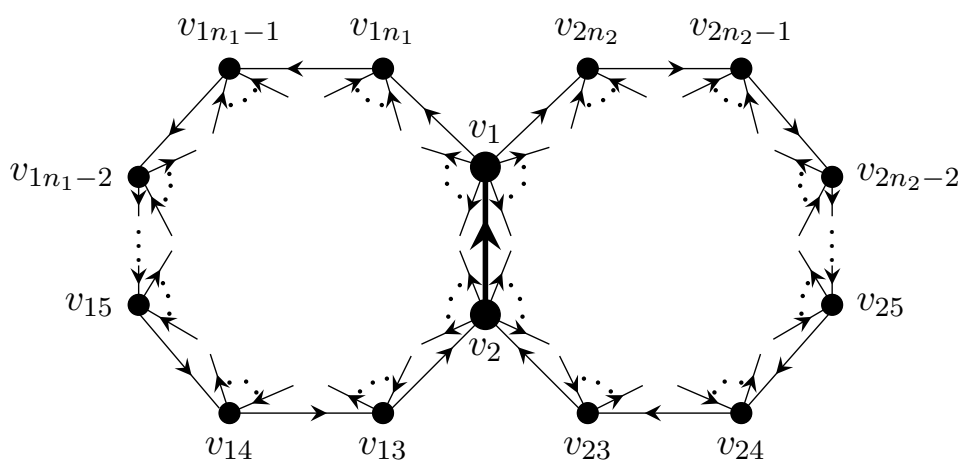

Figure 5. The 2-sum digraph for $D_{n_{1}}$ and $D_{n_{2}}$.

Claim 1. There does not exist a dicycle whose arcs intersect arcs in two or more $D_{n_{i}}$ 's, $(i=1,2, \ldots, s)$.

By Definition 2.3.1, we have $V\left(D_{n_{i}}\right) \cap V\left(D_{n_{j}}\right)=\left\{v_{1}, v_{2}\right\}(i \neq j)$. Without loss of generality, we consider oriented graphs $D_{n_{1}}$ and $D_{n_{2}}$, suppose that there exists a dicycle $C_{0}$ such that

$$
\left\{A\left(C_{0}\right)-\left(v_{2}, v_{1}\right)\right\} \cap A\left(D_{n_{1}}\right) \neq \emptyset \text { and }\left\{A\left(C_{0}\right)-\left(v_{2}, v_{1}\right)\right\} \cap A\left(D_{n_{2}}\right) \neq \emptyset
$$

Thus, there must exist four different arcs

$$
\left\{\left(v_{1 i^{\prime}}, v_{1}\right),\left(v_{1}, v_{2 i^{\prime \prime}}\right),\left(v_{2 j^{\prime \prime}}, v_{2}\right),\left(v_{2}, v_{1 j^{\prime}}\right)\right\} \in A\left(C_{0}\right)
$$

with $\left(v_{1 i^{\prime}}, v_{1}\right),\left(v_{2}, v_{1 j^{\prime}}\right) \in A\left(D_{n_{1}}\right)$ and $\left(v_{1}, v_{2 i^{\prime \prime}}\right),\left(v_{2 j^{\prime \prime}}, v_{2}\right) \in A\left(D_{n_{2}}\right)$, as shown in Figure 6 ; or four different arcs

$$
\left\{\left(v_{1 s^{\prime}}, v_{2}\right),\left(v_{2}, v_{2 s^{\prime \prime}}\right),\left(v_{2 k^{\prime \prime}}, v_{1}\right),\left(v_{1}, v_{1 k^{\prime}}\right)\right\} \in A\left(C_{0}\right)
$$

with $\left(v_{1 s^{\prime}}, v_{2}\right),\left(v_{1}, v_{1 k^{\prime}}\right) \in A\left(D_{n_{1}}\right)$ and $\left(v_{2}, v_{2 s^{\prime \prime}}\right),\left(v_{2 k^{\prime \prime}}, v_{1}\right) \in A\left(D_{n_{2}}\right)$, as shown in Figure 7 .

By the Definition 2.3.1, Lemma 2.2.6 (iii) and (1), we have $N_{D}^{-}\left(v_{1}\right)=\left\{v_{2}\right\}$, and so $v_{1 i^{\prime}}=v_{2}$ or $v_{2 k^{\prime \prime}}=v_{2}$, contrary to the assumption that $C_{0}$ is a dicycle. This proves Claim 1.

By Claim 1, for every dicycle $C$ in $D$, all arcs in $C$ (except for the arc $\left(v_{2}, v_{1}\right)$ ) belong to exactly one of oriented graphs $D_{n_{i}}(i=1,2, \ldots, n)$. By Definition 2.2.5 and Lemma 2.2.7, every dicycle cover of oriented graph $D_{n_{i}}(i=1,2, \ldots, n)$ must have at least $m_{i}-n_{i}+1$ dicycles. This completes the proof. 


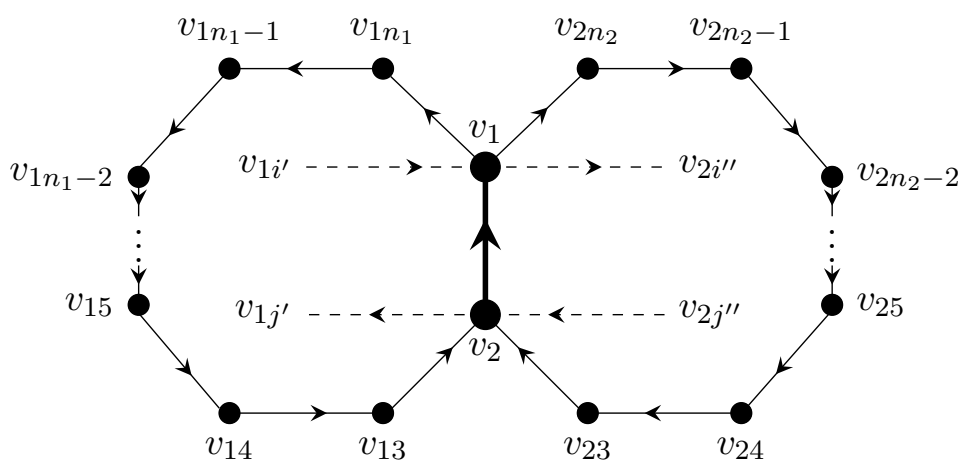

Figure 6

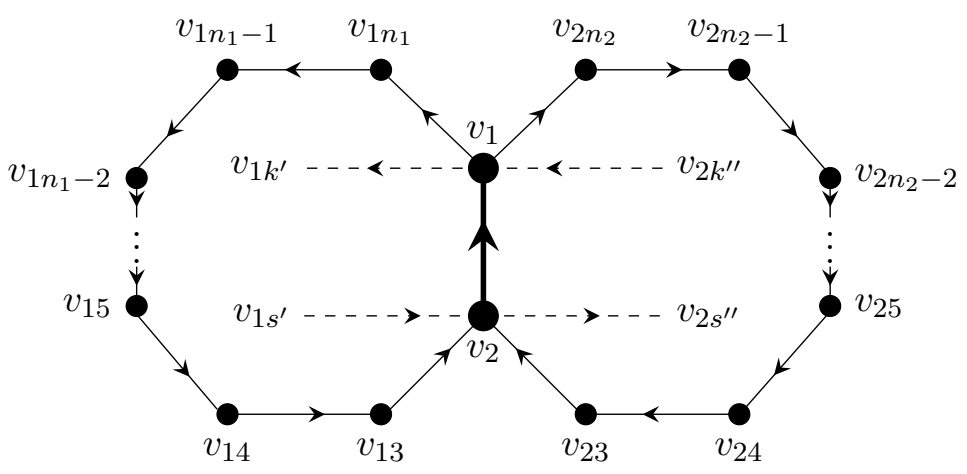

Figure 7

By Corollary 2.2.10 and Theorem 2.3.3, we have the following Corollary.

Corollary 2.3.4. Let $D_{n_{1}}, D_{n_{2}}, \ldots, D_{n_{s}}$ be $s$ disjoint strong tournaments with $n_{1}, n_{2}, \ldots, n_{s}$ vertices, respectively. Then $D_{n_{1}} \oplus_{2} D_{n_{2}} \oplus_{2} \ldots \oplus_{2} D_{n_{s}}$ has a dicycle cover $\mathcal{C}$ with $|\mathcal{C}| \leq\left(\frac{n_{1}\left(n_{1}-1\right)}{2}+\right.$ $\left.\frac{n_{2}\left(n_{2}-1\right)}{2}+\ldots+\frac{n_{s}\left(n_{s}-1\right)}{2}\right)-\left(n_{1}+n_{2}+\ldots+n_{s}\right)+s$. This bound is best possible.

Let $G_{n}$ be a Hamiltonian graph with $n$ vertices and $m$ arcs, let $D_{n}^{i}(i$ is an integer ) denote a Hamiltonian orientation of $G_{n}$. For a positive integer $s$, let $H\left(G_{n}, s\right)$ denote the family of all 2-sum generated digraphs $D_{n}^{1} \oplus_{2} D_{n}^{2} \oplus_{2} \ldots \oplus_{2} D_{n}^{s}$, as well as a member in the family (for notational convenience). By the definition of $H\left(G_{n}, s\right)$, we have $H\left(G_{n}, 1\right)=D_{n}^{1}, H\left(G_{n}, s\right)=$ $H\left(G_{n}, s-1\right) \oplus_{2} D_{n}^{s}$. The conclusions of next corollaries follow from Theorem 2.1.1. The sharpness of these corollaries can be demonstrated using similar constructions displayed in Lemma 2.2.7 and Corollary 2.2.13.

Corollary 2.3.5. Let $m, n \geq 3$ be integer, $G_{n}$ be a Hamiltonian graph with $n$ vertices and $m$ edges, and $K_{n}$ be a complete graph on $n \geq 3$ vertices. 
(i) Any member in $H\left(G_{n}, s\right)$ has a dicycle cover $\mathcal{C}$ with $|\mathcal{C}| \leq s(m-n+1)$. This bound is best possible.

(ii) In particular, any $H\left(K_{n}, s\right)$ has a dicycle cover $\mathcal{C}$ with $|\mathcal{C}| \leq s\left(\frac{n(n-1)}{2}-n+1\right)$. This bound is best possible.

Corollary 2.3.6. Let $m, n \geq 3$ be integer, $B_{n}$ be a Hamiltonian bipartite graph with $2 n$ vertices and $m$ edges, and $K_{n, n}$ be a complete bipartite graph.

(i) Any $H\left(B_{n}, s\right)$ has a dicycle cover $\mathcal{C}$ with $|\mathcal{C}| \leq s(m-2 n+1)$. This bound is best possible.

(ii) In particular, any $H\left(K_{n, n}, s\right)$ has a dicycle cover $\mathcal{C}$ with $|\mathcal{C}| \leq s(n-1)^{2}$. This bound is best possible. 


\section{Chapter 3}

\section{Supereulerian Digraphs with given local structures}

\subsection{Introduction}

We consider finite graphs and digraphs. Undefined terms and notations will follow [1] for graphs and [2] for digraphs. As in [2], $(u, v)$ represents an arc oriented from a vertex $u$ to a vertex $v$. A digraph $D$ is simple if $D$ has no loops and if for any pair of distinct vertices $u, v \in V(D)$, there is at most one arc in $D$ oriented from $u$ to $v$. For an integer $n>0$, we use $K_{n}^{*}$ to denote the complete digraph on $n$ vertices. Hence for every pair of distinct vertices $u, v \in V\left(K_{n}^{*}\right)$, there is exactly one $\operatorname{arc}(u, v)$ in $A\left(K_{n}^{*}\right)$. A cycle on $n$ vertices is often called an $n$-cycle. For a digraph $D$, the underlying graph of $D$, denoted by $G(D)$, is obtained from $D$ by erasing the orientations of all $\operatorname{arcs}$ of $D$. A ditrail in $D$ is an alternating sequence of vertices and arcs such that all the arcs are distinct. If all the vertices of a ditrail are distinct we call it a dipath. An $\operatorname{arc}(u, v) \in A(D)$ is symmetric in $D$ if $(u, v),(v, u) \in A(D)$. A digraph $D$ is symmetric if $|V(D)|=1$ or if $|A(D)|>0$ and every arc of $D$ is symmetric. Especially, a symmetric dipath $P$ is a dipath such that every arc of $P$ is symmetric. Following [2], if $X, Y \subseteq V(D)$, then define

$$
(X, Y)_{D}=\{(x, y) \in A(D): x \in X, y \in Y\} .
$$

When $Y=V(D)-X$, we define

$$
\partial_{D}^{+}(X)=(X, V(D)-X)_{D} \text { and } \partial_{D}^{-}(X)=(V(D)-X, X)_{D}
$$

If $X \subseteq V(D) \cup A(D)$, then $D[X]$ denotes the subdigraph induced by $X$. If $S$ is a subdigraph of a digraph $D$ and if $X \subset A(S)$ and $Y \subseteq A(D)-A(S)$, we use $S-X+Y$ to denote $D[(A(S)-X) \cup Y]$. 
For a vertex $v \in V(D)$, the out-degree of $v$ is $d_{D}^{+}(v)=\left|\partial_{D}^{+}(\{v\})\right|$ and the in-degree of $v$ in $D$ is $d_{D}^{-}(v)=\left|\partial_{D}^{-}(\{v\})\right|$. Furthermore, we define

$$
\partial_{D}(v)=\partial_{D}^{+}(\{v\}) \cup \partial_{D}^{-}(\{v\}) \text { and } d_{D}(v)=d_{D}^{+}(v)+d_{D}^{-}(v)
$$

Let $N_{D}^{+}(v)=\{u \in V(D):(v, u) \in A(D)\}$ and $N_{D}^{-}(v)=\{u \in V(D):(u, v) \in A(D)\}$ denote the out neighbors and in neighbors of $v$ in $D$, respectively. When the digraph $D$ is understood from the context, we often omit the subscript $D$. Following [1] and [2], we use $\lambda(D)$ and $c(D)$ to denote the arc-strong connectivity of $D$ and the number of components of the underlying graph $G(D)$ of $D$, respectively. By the definition of $\lambda(D)$ in [2], for any integer $k \geq 0$,

$$
\lambda(D) \geq k \text { if and only if for any nonempty proper subset } X \subset V(D),\left|\partial_{D}^{+}(X)\right| \geq k \text {. }
$$

Boesch, Suffel, and Tindell [30] in 1977 proposed the supereulerian problem, which seeks to characterize graphs that have spanning eulerian subgraphs, and they indicated such that problem would be very difficult. Pulleyblank [26] later in 1979 proved that determining whether a graph is supereulerian, even within planar graphs, is NP-complete. Since then, there have been lots of researches on this topic. Catlin [20] in 1992 presented the first survey on supereulerian graphs. Later Chen et al [21] gave an update in 1995, specifically on the reduction method associated with the supereulerian problem. A recent survey on supereulerian graphs is given in $[24]$.

It is natural to consider the supereulerian problem in digraphs. A strong digraph $D$ is eulerian if for each vertex $v \in V(D)$, we have $d_{D}^{+}(v)=d_{D}^{-}(v)$. A strong digraph $D$ is supereulerian if $D$ contains a spanning eulerian subdigraph. One of the central problems is to characterize supereulerian digraphs.

Theorem 3.1.1. (Jaeger [23] and Catlin [19]) Every 4-edge-connected graph is supereulerian.

Theorem 3.1.2. (Catlin, Corollary 1 of [19]) there exist graph families $\mathcal{F}$ such that if every edge of a connected graph $G$ lies in a subgraph of $G$ isomorphic to a member in $\mathcal{F}$, then $G$ is supereulerian. In particular, if every edge of $G$ lies in a 3-cycle of $G$, then $G$ is supereulerian.

The purpose of this paper is to see if the above-mentioned results of Jaeger and Catlin can be extended to digraphs. Firstly, we shall show that both Theorem 3.1.1 and Theorem 3.1.2 cannot be directly extended to digraphs. To be more precise, we in the next section will show that for any integer $k>0$, there exists infinitely many non-supereulerian digraphs $D$ with $\lambda(D) \geq k$. To show that Theorem 3.1.2 cannot be extended to digraphs, we need more concepts. A digraph $D$ is weakly connected if $G(D)$, its underlying graph, is connected. Let $\mathcal{H}$ be a 
family of digraphs. A strong digraph $D$ is locally $\mathcal{H}$ if every arc $a \in A(D)$ lies in a subdigraph $H_{a}$ of $D$, where $H_{a} \in \mathcal{H}$. For convenience, we also call a locally $\{H\}$ digraph $D$ as a locally $H$ digraph. Let $C_{3}$ denote a directed 3-cycle. Unlike graphs, we in Section 2 will also show that a strong and locally $C_{3}$ digraph may not be supereulerian. Thus what local structures will assure supereulerian property will be the objective of this research. We will introduce the graph families of symmetrically connected digraphs and partially symmetric digraphs, in subsequent sections below, and prove the following results.

Theorem 3.1.3. Each of the following holds.

(i) Every symmetrically connected digraph is supereulerian.

(ii) Every partially symmetric digraph is supereulerian.

Moreover, in the Sections 3 and 4, we will show that every weakly connected locally symmetrically connected digraph is supereulerian and every weakly connected locally partially symmetric digraph is supereulerian. The sharpness of these results are also discussed.

\subsection{Examples of nonsupereulerian strong digraphs}

As Catlin in [19] indicated that

every connected graph in which every edge lies in a 3-cycle is supereulerian,

it is natural to see if every strong digraph in which every arc lies in a directed 3-cycle is supereulerian. In this section, we shall present, for any integer $k>0$, an infinite family of $\mathcal{D}$ such that every digraph in $\mathcal{D}$ is locally $\left\{C_{3}\right\}$ with $\lambda(D) \geq k$ but nonsupereulerian.

We need the following necessary condition for a digraph to be supereulerian. Let $D$ be a digraph and $U \subset V(D)$. We call a collection of ditrails $P_{1}, P_{2}, \cdots, P_{t}$ of the induced subdigraph $D[U]$ a cover of $U$ if $\cup_{i=1}^{t} V\left(P_{i}\right)=U$ and $A\left(P_{i}\right) \cap A\left(P_{j}\right)=\emptyset$, whenever $i \neq j$. The minimum value of such $t$ is denoted by $\tau(U)$. For any subset $A \subseteq V(D)-U$, define $B=: V(D)-U-A$. Let

$$
h(U, A)=: \min \left\{\left|\partial_{D}^{+}(A)\right|,\left|\partial_{D}^{-}(A)\right|\right\}+\min \left\{\left|(U, B)_{D}\right|,\left|(B, U)_{D}\right|\right\}-\tau(U) .
$$

Then we have the following proposition.

Proposition 3.2.1. (Hong, Lai and Liu, Proposition 2.1 of [34]) If D has a spanning eulerian subdigraph, then for any $U \subset V(D)$, and for any subset $A \subseteq V(D)-U$, we have $h(U, A) \geq 0$. 
The authors in [17] and [34] have independently presented infinite families of non supereulerian digraphs with arbitrarily high arc-strong connectivity. In those digraphs shown in [17] and [34], there exist some arcs which are not lying in a directed 3-cycle. In this section, we will construct an infinite family of non supereulerian digraphs with arbitrarily high arc-strong connectivity such that every arc of each of these digraphs lies in a directed 3-cycle.

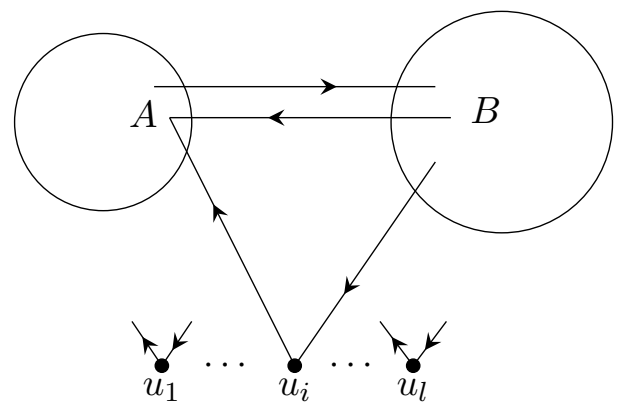

Figure 1. The digraph $D=D(\alpha, \beta, k, \ell)$.

Example 3.2.2. Let $\alpha, \beta, k>0$ be integers with $\alpha, \beta \geq k+1$, and let $A$ and $B$ be two disjoint set of vertices with $|A|=\alpha$ and $|B|=\beta$. Let $\ell \geq \alpha \beta+1$ be an integer, and $U$ be a set of vertices disjoint from $A \cup B$ with $|U|=\ell$. We construct a digraph $D=D(\alpha, \beta, k, \ell)$ such that $V(D)=A \cup B \cup U$ and the arcs of $D$ are given as required in (D1) and (D2) below. (See Figure 1).

(D1) $D[A \cup B] \cong K_{\alpha+\beta}^{*}$ is a complete digraph.

(D2) For every vertex $u \in U$, and for every $v \in A,(u, v) \in A(D)$ and for every $w \in B$, $(w, u) \in A(D)$. Thus for any $u \in U$, we have $N_{D}^{+}(u)=A$ and $N_{D}^{-}(u)=B$. No two vertices in $U$ are adjacent.

A digraph $D$ is quasitransitive if, for every triple of distinct vertices $x, y, z \in V(D)$, with $(x, y),(y, z) \in A(D)$, there is at least one arc between $x$ and $z$. Thus the digraphs in Example 3.2.2 are quasitransitive. Using canonical decompositions of quasitransitive digraphs by Bang-Jensen and Huang in [16], Bang-Jensen and Maddaloni characterized supereulerian quasitransitive digraphs and further showed that there exists a polynomial algorithm to determine if a quasitransitive digraph is supereulerian in [17].

Proposition 3.2.3. Let $D=D(\alpha, \beta, k, \ell)$ for some given parameters $\alpha, \beta, k$ and $\ell$ as defined in Example 3.2.2. Then each of the following holds.

(i) $\lambda(D)>k$.

(ii) Every arc of D lies in a directed 3-cycle.

(iii) $D$ is not supereulerian. 
Proof. (i) We use (3.1) to show (i). Let $\emptyset \neq X \subset V(D)$ be a proper nonempty subset. Let $n_{X}=|X \cap(A \cup B)|$. If $0<n_{X}<\alpha+\beta$, then by Example 3.2.2(D1), $\partial_{D}^{+}(X) \geq \mid(X \cap U, A-$ $X)_{D} \cup(X \cap(A \cup B),(A \cup B)-X)_{D} \mid \geq n_{X}\left(\alpha+\beta-n_{X}\right) \geq \alpha+\beta-1 \geq 2 k+1>k$. Hence we assume that either $A \cup B \subseteq X$ or $X \cap(A \cup B)=\emptyset$. If $X \cap(A \cup B)=\emptyset$, then $X \subseteq U$, and so by Example 3.2.2(D2), $\left|\partial_{D}^{+}(X)\right| \geq\left|(X, A)_{D}\right| \geq|A|>k$. Thus we may assume that $A \cup B \subseteq X$. Then $\left|\partial_{D}^{+}(X)\right|=\left|(B, U-X)_{D}\right| \geq|B|>k$. Hence by $(1.1), \lambda(D)>k$.

(ii) Let $a=(u, v)$ be an $\operatorname{arc}$ in $D$. If $u, v \in A \cup B$, then by Example 3.2.2(D1), $a$ is in a $K_{\alpha+\beta}^{*}$ and so $a$ lies in a directed 3 -cycle of $D$. Since $U$ is an independent set of $D$, by Example 3.2.2(D2), we may assume that $u \in B$ and $v \in U$, whence for any $w \in A$, wuvw is a directed 3-cycle; if $u \in U$ and $v \in A$, then for any $w^{\prime} \in B, w^{\prime} u v w^{\prime}$ is a directed 3-cycle. This justifies (ii).

(iii) We apply Proposition 3.2.1. By (D1), $D[A \cup B] \cong K_{\alpha+\beta}^{*}$, and so $\left|\partial_{D}^{+}(A)\right|=\alpha \beta$. By (D2), $\left|(U, B)_{D}\right|=0$ and so $\tau(U)=|U|>\alpha \beta$. It follows that

$$
h(U, A)=\left|\partial_{D}^{+}(A)\right|+\left|(U, B)_{D}\right|-\tau(U)=\alpha \beta-|U|<0 .
$$

It follows from Proposition 3.2.1 that $D$ is not supereulerian.

Thus Example 3.2.2 indicates that there exists an infinite family of non supereulerian digraphs with arbitrarily high arc-strong connectivity such that every arc of each of these digraphs lies in a directed 3-cycle. Hence both Theorem 3.1.1 and Theorem 3.1.2 cannot be directly extended to digraphs.

\subsection{Locally symmetrically connected supereulerian digraphs}

In this section, we will introduce symmetrically connected digraphs and show that every locally symmetrically connected digraph is supereulerian. We will also show that this result is best possible in some sense.

Definition 3.3.1. Let $D$ be a digraph such that either $D=K_{1}$ or $A(D) \neq \emptyset$. If for any $u, v \in V(D), D$ contains a symmetric dipath from $u$ to $v$, then $D$ is called a symmetrically connected digraph. Let $\mathcal{S C}$ be the family of all symmetrically connected digraphs.

Theorem 3.3.2. Every symmetrically connected digraph is supereulerian.

Proof. By contradiction, we assume that $D$ is not supereulerian. By Definition 3.3.1 and (3.1), $D$ is strong. Thus $D$ contains a nontrivial eulerian subdigraph. Choose $S$ to be an eulerian 
subdigraph of $D$ such that

$$
|V(S)| \text { is maximized among all eulerian subdigraph of } D \text {. }
$$

Since $D$ is not supereulerian, we have $|V(S)|<|V(D)|$. Pick a vertex $u \in V(D)-V(S)$ and $v \in$ $V(S)$. As $u, v \in V(D)$, and $D$ is symmetrically connected, $D$ contains a symmetric dipath $P=$ $v_{0} v_{1} \cdots v_{m}$ with $v_{0}=u$ and $v_{m}=v$. Since $u \in V(D)-V(S)$ and $v \in V(S)$, there exists a smallest integer $t>0$ such that $v_{t} \in V(S)$. Since $P$ is symmetric, the $\operatorname{arcs}\left(v_{1}, v_{0}\right),\left(v_{2}, v_{1}\right), \cdots,\left(v_{t}, v_{t-1}\right) \in$ $A(D)$. It follows that $D\left[A(S) \cup\left\{\left(v_{0}, v_{1}\right),\left(v_{1}, v_{2}\right), \cdots,\left(v_{t-1}, v_{t}\right),\left(v_{1}, v_{0}\right),\left(v_{2}, v_{1}\right), \cdots,\left(v_{t}, v_{t-1}\right)\right\}\right]$ is also an eulerian subdigraph of $D$, contrary to (3.3).

The symmetric difference between two digraphs $D_{1}$ and $D_{2}$, written as $D_{1} \triangle D_{2}$, is the induced digraph on the arc set $\left(A\left(D_{1}\right) \cup A\left(D_{2}\right)\right)-\left(A\left(D_{1}\right) \cap A\left(D_{2}\right)\right)$. Thus $D_{1} \triangle D_{2}$ has no isolated vertices and contains arcs that are either in $D_{1}$ or in $D_{2}$ but not in both.

Corollary 3.3.3. Every weakly connected locally $\mathcal{S C}$ digraph is supereulerian.

Proof. Let $D$ be a weakly connected and locally symmetrically connected digraph. We will prove that $D$ is symmetrically connected. For any $u_{1}, u_{k} \in V(D)$, we shall follow Definition 3.3 .1 to show there exists a symmetric dipath from $u_{1}$ to $u_{k}$. Since $D$ is weakly connected then there exists an undirected path $P$ in $G(D)$ lying between $u_{1}$ and $u_{k}$. If $P$ has only one arc and since $\left\{\left(u_{1}, u_{k}\right),\left(u_{k}, u_{1}\right)\right\} \cap A(D) \neq \emptyset$, by Definition 3.3.1, $\left\{u_{1}, u_{k}\right\}$ are endpoints of a symmetrically connected dipath $P$. Then $D$ contains a symmetric dipath from $u_{1}$ to $u_{k}$ and we are done. If not, then for any continuous three vertices $\left\{u_{j-1}, u_{j}, u_{j+1}\right\} \in V(P)$ where $1 \leq j-1$ and $j+1 \leq k$. Since $\left\{\left(u_{j-1}, u_{j}\right),\left(u_{j}, u_{j-1}\right)\right\} \cap A(D) \neq \emptyset$ and $\left\{\left(u_{j}, u_{j+1}\right),\left(u_{j+1}, u_{j}\right)\right\} \cap A(D) \neq \emptyset$, by Definition 3.3.1, $\left\{u_{j-1}, u_{j}\right\}$ are endpoints of a symmetrically connected dipath $P_{1}$ and $\left\{u_{j}, u_{j+1}\right\}$ are endpoints of a symmetrically connected dipath $P_{2}$. Since $u_{j} \in V\left(P_{1}\right) \cap V\left(P_{2}\right)$, implies that $V\left(P_{1}\right) \cap V\left(P_{2}\right) \neq \emptyset$. Then there exists a symmetrically connected dipath $P_{3} \subseteq\left(P_{1} \triangle P_{2}\right)$ with endpoints $u_{j-1}$ and $u_{j+1}$. Then there exists a symmetrically connected subdigraph lies between $u_{j-1}$ and $u_{j+1}$. This shows that the locally symmetrically connected digraph $D$ is transitive for the arcs of any undirected path. Hence, by transitivity, $\left(u_{1}, u_{k}\right)$ lies in a symmetrically connected subdigraph. This shows that $D$ contains a symmetric dipath from $u_{1}$ to $u_{k}$ implies that $D$ is symmetrically connected. By Theorem 3.3.2, $D$ is supereulerian.

The sharpness of Corollary 3.3.3 will be justified in the proposition below. 
Proposition 3.3.4. Let $H$ be a strong digraph with $|V(H)|=n>1$. If there exists a vertex $v \in V(H)$ such that $d(v) \leq n-1$ and $v$ is not incident with any symmetric arcs, then there exists an infinite family $\mathcal{F}(H)$ of strong, locally $H$, non-supereulerian digraphs.

Proof. Let $H$ be such a given digraph. Then $H$ contains a vertex $v$ such that $v$ is not incident with any symmetric arcs. We have $N_{H}^{+}(v) \cap N_{H}^{-}(v)=\emptyset$.

Let $\alpha \geq\left|N_{H}^{+}(v)\right|$ and $\beta \geq\left|N_{H}^{-}(v)\right|$ be integers such that $\alpha+\beta \geq n$, and let $k=1$, and $\ell \geq \alpha \beta+1$ be integers. Define $D=D(\alpha, \beta, k, \ell)$ as in Example 3.2.2. We have the following observations, which justify the proposition.

(A) $D$ is strong and nonsupereulerian. This observation follows from Proposition 3.2.3 with $k=1$.

(B) For any arc $a \in A(D)$, there exists a subdigraph $H_{a}$ of $D$ such that $H_{a}$ is isomorphic to $H$.

Let $a=(x, y)$ be an arc in $A(D)$. If $a \in A(D[A \cup B])$, then by (D1), $D[A \cup B] \cong K_{\alpha+\beta}^{*}$. It follows by the assumption that $\alpha+\beta \geq n=|V(H)|$ that $D[A \cup B]$ has a subdigraph isomorphic to $H$ which contains $a$. Hence by (D2), we may assume that $(x, y) \in(A \cup B, U)_{D}$ or $(x, y) \in$ $(U, A \cup B)_{D}$. In either case, let $A^{\prime} \subseteq A$ and $B^{\prime} \subseteq B$ be subsets with $\left|A^{\prime}\right|=\left|N_{H}^{+}(v)\right|$ and $\left|B^{\prime}\right|=\left|N_{H}^{-}(v)\right|$, respectively. Then $D\left[A^{\prime} \cup B^{\prime} \cup\{x, y\}\right]$ contains a subdigraph isomorphic to $H$ and contains $(x, y)$. This proves $(\mathrm{B})$ and justifies that $D$ is locally $H$.

\subsection{Partially symmetric supereulerian digraphs}

We investigate a different kind of local structural condition which warrants a digraph to be supereulerian. For any digraph $D$, define a relation on $V(D)$ such that $u \sim v$ if and only if $u=v$ or $D$ has a symmetrically connected subdigraph $H$ with $u, v \in V(H)$. If $H_{1}$ and $H_{2}$ are two subdigraphs of $D$, then we define $H_{1} \cup H_{2}$ to be the subdigraph of $D$ with vertex set $V\left(H_{1}\right) \cup V\left(H_{2}\right)$ and arc set $A\left(H_{1}\right) \cup A\left(H_{2}\right)$.

Lemma 3.4.1. If $H_{1}$ and $H_{2}$ are two symmetrically connected subdigraphs of $D$ such that $V\left(H_{1}\right) \cap V\left(H_{2}\right) \neq \emptyset$, then $H_{1} \cup H_{2}$ is also a symmetrically connected subdigraph of $D$.

Proof. It suffices to show that for any $u, v \in V\left(H_{1} \cup H_{2}\right), H_{1} \cup H_{2}$ contains a symmetric dipath from $u$ to $v$. If $u, v \in V\left(H_{1}\right)$, then since $H_{1}$ is symmetrically connected, by Definition 3.3.1, $H_{1}$ contains a symmetric dipath from $u$ to $v$. Hence $H_{1} \cup H_{2}$ has a symmetric dipath from $u$ to $v$. Similarly, If $u, v \in V\left(H_{2}\right)$, then $H_{1} \cup H_{2}$ also has a symmetric dipath from $u$ to $v$. 
Thus we assume that $u \in V\left(H_{1}\right) \backslash V\left(H_{2}\right)$ and $v \in V\left(H_{2}\right) \backslash V\left(H_{1}\right)$. Since $V\left(H_{1}\right) \cap V\left(H_{2}\right) \neq \emptyset$, we can take a vertex $r \in V\left(H_{1}\right) \cap V\left(H_{2}\right)$. Since $H_{1}$ and $H_{2}$ are symmetrically connected, $H_{1}$ contains a $(u, r)$ - symmetric dipath $P_{1}$ and $H_{2}$ contains a $(r, v)$-symmetric dipath $P_{2}$. It follows that $D\left[A\left(P_{1}\right) \cup A\left(P_{2}\right)\right]$ contains a symmetric dipath from $u$ to $v$.

By Lemma 3.4.1, the relation $\sim$ is an equivalence relation on $V(D)$. Each equivalence class induces a maximal symmetrically connected subdigraph of $D$. We have the following observation.

Observation 3.4.2. Let $D$ be a digraph. Each of the following holds.

(i) D has a unique collection of maximal symmetrically connected subdigraphs.

(ii) If $H_{1}$ and $H_{2}$ are two maximal symmetrically connected subdigraphs, then either $H_{1}=H_{2}$, or $V\left(H_{1}\right) \cap V\left(H_{2}\right)=\emptyset$.

Definition 3.4.3. Let $c \geq 2$ be an integer and let $D$ be a weakly connected digraph and let $\left\{H_{1}, H_{2}, \cdots, H_{c}\right\}$ be the set of maximal symmetrically connected subdigraphs of $D$.

(i) If for any proper nonempty subset $\mathcal{J} \subset\left\{H_{1}, H_{2}, \cdots, H_{c}\right\}$, there exist an $H_{i} \in \mathcal{J}$ and a vertex $v \in V\left(H_{i}\right)$, and an $H_{j} \notin \mathcal{J}$ such that

$$
N_{D}^{+}(v) \cap V\left(H_{j}\right) \neq \emptyset \text { and } N_{D}^{-}(v) \cap V\left(H_{j}\right) \neq \emptyset,
$$

then $D$ is partially symmetric.

(ii) Let $\mathcal{P S}$ denote the family of all partially symmetric digraphs.

For a digraph $D$ and let $\left\{H_{1}, H_{2}, \cdots, H_{c}\right\}$ be the set of all symmetrically connected components of $D$. Define $D^{\prime}$ to be the digraph obtained from $D$ by contracting all symmetrically connected components. By Definition 3.4.3, $D$ is partially symmetric if and only if $D^{\prime}$ is symmetrically connected. In fact, if $D^{\prime}$ is symmetrically connected, then Definition 3.4.3 (i) holds. Conversely, let $M$ be a symmetrically connected component of $D^{\prime}$. If $M \neq D^{\prime}$, then $\mathcal{J}=V(M)$ is a subset of all symmetrically connected components of $D$, and so by Definition 3.4.3(i), there exists a vertex $H_{i} \in V(M)$ and a $H_{j} \in V\left(D^{\prime}\right)-V(M)$ such that both $\left(H_{i}, H_{j}\right),\left(H_{j}, H_{i}\right) \in A\left(D^{\prime}\right)$, contrary to the assumption that $M$ is a component.

Let $K_{s}^{*}$ and $K_{t}^{*}$ be two complete digraphs of order $s \geq 2$ and $t \geq 2$, respectively, such that $u_{1}, u_{2}$ are two distinct vertices of $K_{s}^{*}$ and $v_{1}, v_{2}$ are two distinct vertices of $K_{t}^{*}$. Let $D$ be the digraph obtained from the disjoint union of $K_{s}^{*}$ and $K_{t}^{*}$ by adding the $\operatorname{arcs}\left(u_{1}, v_{1}\right)$ and $\left(v_{2}, u_{2}\right)$. Then by the remark above, $D$ is partially symmetric but not symmetrically connected.

Example 3.4.4. Let $J_{1}, J_{2}$ be digraphs with $V\left(J_{1}\right)=V\left(J_{2}\right)=\left\{v_{1}, v_{2}, v_{3}, v_{4}\right\}$ and $A\left(J_{1}\right)=$ $\left\{\left(v_{1}, v_{2}\right),\left(v_{2}, v_{1}\right)\right.$ 
$\left.\left(v_{1}, v_{4}\right),\left(v_{2}, v_{3}\right),\left(v_{3}, v_{4}\right),\left(v_{4}, v_{3}\right),\left(v_{3}, v_{1}\right),\left(v_{4}, v_{2}\right)\right\}$, and $A\left(J_{2}\right)=\left\{\left(v_{1}, v_{2}\right),\left(v_{2}, v_{1}\right),\left(v_{1}, v_{3}\right),\left(v_{1}, v_{4}\right),\left(v_{3}, v_{4}\right)\right.$, $\left.\left(v_{4}, v_{3}\right),\left(v_{3}, v_{2}\right),\left(v_{4}, v_{2}\right)\right\}$. See Figure 2. Then we have these observations.

(i) For $i \in\{1,2\}$, $J_{i}$ does not have a symmetric $\left(v_{2}, v_{3}\right)$-dipath, and so $J_{i}$ is not symmetrically connected.

(ii) For $i \in\{1,2\}$, the maximal symmetrically connected subdigraph of $J_{i}$ are $H_{1}=D\left[\left\{v_{1}, v_{2}\right\}\right]$ and $H_{2}=D\left[\left\{v_{3}, v_{4}\right\}\right]$.

(iii) By Definition 3.4.3(i), $J_{1}$ is partially symmetric but $J_{2}$ is not partially symmetric.

(iv) For any arc $a \in A\left(K_{4}^{*}\right)-A\left(J_{2}\right), J_{2}+a$ is symmetrically connected.
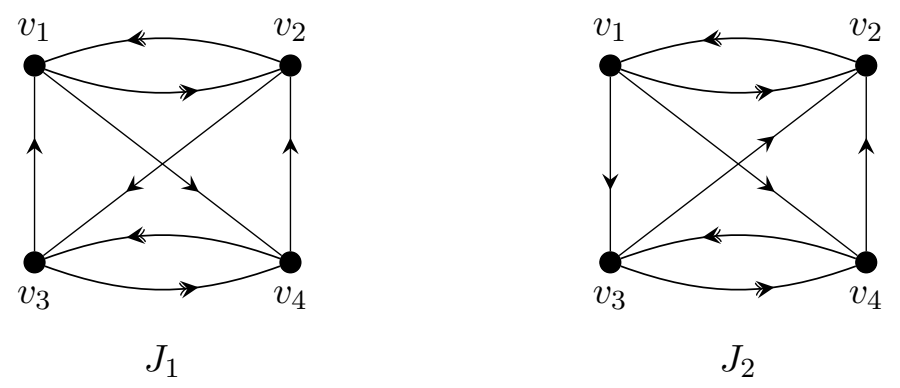

Figure $2: J_{1}$ is partially symmetric digraph but $J_{2}$ is not partially symmetric digraph.

Lemma 3.4.5. Let $D$ be a partially symmetric digraph. Then each of the following holds.

(i) For every $1 \leq i \leq c$, we have that $\left|V\left(H_{i}\right)\right| \geq 2$.

(ii) $D$ is strong.

(iii) $|V(D)| \geq 4$.

(iv) $D$ does not have symmetric arcs connecting a vertex in $H_{i}$ and a vertex in $H_{j}$ for any distinct $i, j \in\{1,2, \cdots, c\}$.

Proof. Let $\left\{H_{1}, H_{2}, \cdots, H_{c}\right\}$ be the set of maximal symmetrically connected subdigraphs of $D$ with $c \geq 2$.

(i) By contradiction, we assume that for some maximal symmetrically connected subdigraph $H_{i}$ of $D, V\left(H_{i}\right)=\{u\}$. Let $\mathcal{J}=\left\{H_{1}, H_{2}, \cdots, H_{c}\right\}-\left\{H_{i}\right\}$. Since $D$ is partially symmetric, there must be an $H_{j} \in \mathcal{J}$ and a vertex $v \in V\left(H_{j}\right)$ such that $(v, u),(u, v) \in A(D)$. It follows by the maximality of $H_{j}$ that $u \in V\left(H_{j}\right)$, contrary to Observation 3.4.2 (ii). Hence Lemma 3.4.5(i) must hold.

(ii) Let $X$ be a nonempty proper subset of $V(D)$. By (3.1), it suffices to show that $\left|\partial_{D}^{+}(X)\right| \geq 1$. If for some $i$, both $X \cap V\left(H_{i}\right) \neq \emptyset$ and $V\left(H_{i}\right)-X \neq \emptyset$, then by Definition 3.3.1, $\left|\partial_{D}^{+}(X)\right| \geq$ 
$\left|\partial_{H_{i}}^{+}\left(X \cap V\left(H_{i}\right)\right)\right| \geq 1$. Hence we assume that no such $H_{i}$ exists. Since $V(D)=\cup_{i=1}^{c} V\left(H_{i}\right)$, we may assume that for some integer $m$ with $1 \leq m<c, X=\cup_{j=1}^{m} V\left(H_{j}\right)$. Since $D$ is partially symmetric, by Definition 3.4.3, there exist a vertex $x \in V\left(H_{h}\right)$ for some $1 \leq h \leq m$ and a maximal symmetrically connected subdigraph $H_{r}$ with $m+1 \leq r \leq c$ such that $N_{D}^{+}(x) \cap V\left(H_{r}\right) \neq$ $\emptyset$ and $N_{D}^{-}(x) \cap V\left(H_{r}\right) \neq \emptyset$. This implies that $\left|\partial_{D}^{+}(X)\right| \geq 1$, and so $D$ must be strong.

(iii) By Definition 3.4.3 we have that $c \geq 2$, and by (i) each maximal symmetrically connected subdigraph $H_{i}$ with $\left|V\left(H_{i}\right)\right| \geq 2$, this shows that $|V(D)| \geq 4$.

(iv) If for some $i, j \in\{1,2, \cdots, c\}, D$ has symmetric arcs connecting a vertex in $H_{i}$ and a vertex in $H_{j}$, then $H_{i} \cup H_{j}$ is symmetrically connected. By Definition 3.4.3, contrary to $H_{i}$ is maximal symmetrically connected. Hence Lemma 3.4.5(iv) must hold.

Theorem 3.4.6. Every partially symmetric digraph is supereulerian.

Proof. We argue by contradiction and assume that $D$ is partially symmetric and

$$
D \text { is not supereulerian. }
$$

Let $\left\{H_{1}, H_{2}, \cdots, H_{c}\right\}$ be the set of all maximal symmetrically connected subdigraphs of $D$. Since $D$ is partially symmetric digraph, by Lemma 3.4 .5 (ii), $D$ is strong and so $D$ contains a nontrivial eulerian subdigraph. Choose an eulerian subdigraph $S$ of $D$ such that

$$
|V(S)| \text { is maximized among all eulerian subdigraphs of } D \text {. }
$$

Since $D$ is not supereulerian, $V(D)-V(S) \neq \emptyset$. Since $D$ is strong, there exists an $\operatorname{arc}(u, v) \in$ $\partial_{D}^{+}(V(S))$.

If for some $i$ with $1 \leq i \leq c, u, v \in V\left(H_{i}\right)$. Since $H_{i}$ is symmetrically connected, by Definition 3.3.1, $H_{i}$ has a $(v, u)$-dipath $P=v_{1} v_{2} \cdots v_{k}$ with $v=v_{1}$ and $u=v_{k}$ such that $P$ is symmetric. Since $v_{1}=v \notin V(S)$ and $v_{k}=u \in V(S)$, there exists a smallest index $i_{0}>1$ such that $v_{i_{0}} \in V(S)$. It follows that $D\left[V(S) \cup\left\{v_{i_{0}-1}, v_{i_{0}}\right\}\right]$ is eulerian with one more vertex than $S$, contrary to $(3.5)$.

Therefore, there does not exist such $H_{i}$, consequently, for each maximal symmetrically connected subdigraph $H_{i}$ of $D$, either $V\left(H_{i}\right) \cap V(S)=\emptyset$ or $V\left(H_{i}\right) \subseteq V(S)$. Without loss of generality, we assume that for some $t$ with $1 \leq t \leq c, H_{1}, H_{2}, \cdots, H_{t}$ are contained in $S$ and $H_{t+1} \cdots H_{c}$ are disjoint from $V(S)$. 
Since $D$ is partially symmetric digraphs, by Definition3.4.3(i), there exist a vertex $x \in V\left(H_{k}\right)$ for some $k$ with $1 \leq k \leq t$ and for some $j$ with $t+1 \leq j \leq c$ such that $N_{D}^{+}(x) \cap V\left(H_{j}\right) \neq \emptyset$ and $N_{D}^{-}(x) \cap V\left(H_{j}\right) \neq \emptyset$. Suppose that $x^{\prime} \in N_{D}^{+}(x) \cap V\left(H_{j}\right)$ and $x^{\prime \prime} \in N_{D}^{-}(x) \cap V\left(H_{j}\right)$. Since $H_{j}$ is strong, $H_{j}$ has a $\left(x^{\prime}, x^{\prime \prime}\right)$-dipath $x_{1} x_{2} \cdots x_{q}$ with $x^{\prime}=x_{1}$ and $x^{\prime \prime}=x_{q}$. Since $V\left(H_{j}\right) \cap V(S)=\emptyset$, it follows that $C=D\left[A(P) \cup\left\{\left(x, x^{\prime}\right),\left(x^{\prime \prime}, x\right)\right\}\right]$ is a dicycle of $D-A(S)$. Thus $S^{\prime}=D[A(S) \cup A(C)]$ is also an eulerian subdigraph of $D$ with $\left|V\left(S^{\prime}\right)\right| \geq|V(S)|+1$, contrary to (3.5).

Corollary 3.4.7. Every weakly connected locally $\mathcal{P S}$ digraph is supereulerian.

Proof. Let $D$ be a weakly connected and locally partially symmetric digraph, $\left\{H_{1}, H_{2}, \cdots, H_{c}\right\}$ be the set of maximal symmetrically connected subdigraphs of $D$ with $c \geq 2$. We shall verify Definition 3.4.3(i) to prove that $D$ is partially symmetric.

Let $\mathcal{J}=\left\{H_{i_{1}}, H_{i_{2}}, \cdots, H_{i_{m}}\right\}$ and let $\mathcal{J}^{\prime}=\left\{H_{i_{m+1}}, H_{i_{m+2}}, \cdots, H_{i_{m+s}}\right\}$ with $m+s=c$. Let $X=\cup_{j=1}^{m} V\left(H_{i_{j}}\right)$ and $Y=\cup_{k=1}^{s} V\left(H_{i_{m+k}}\right)$. Since $D$ is weakly connected then there exists an arc $a \in(X, Y)_{D} \cup(Y, X)_{D}$. By Lemma 3.4.5(iv), we may assume that

$D$ does not have any symmetric dipath connecting a vertex in $X$ and a vertex in $Y$.

Since $D$ is locally partially symmetric, by Definition 3.4.3(ii), $D$ contains a partially symmetric subdigraph $Q$ of $D$ with $a \in A(Q)$. Without loss of generality, let $a=(u, v) \in(X, Y)_{D}$. Since $a \in A(Q)$, we have $u \in V(Q) \cap V(X)$ and $v \in V(Q) \cap V(Y)$. Let $\left\{Q_{1}, Q_{2}, \cdots, Q_{d}\right\}$ be the set of maximal symmetrically connected subdigraphs of $Q$ with $d \geq 2$. By (3.6) and by Definition 3.4.3(i), we may assume that for some index $l$ with $1 \leq l \leq d-1$, we have $\cup_{r=1}^{l} V\left(Q_{i_{r}}\right) \subseteq X$ and $\cup_{r=l+1}^{d} V\left(Q_{i_{r}}\right) \subseteq Y$. Since $Q$ is partially symmetric, by Definition 3.4.3(i), for some $h$ with $1 \leq h \leq l$, there exists an $x^{\prime} \in V\left(Q_{i_{h}}\right)$, and for some $k$ with $l+1 \leq k \leq d$, we have $N_{Q}^{+}\left(x^{\prime}\right) \cap V\left(Q_{i_{k}}\right) \neq \emptyset$ and $N_{Q}^{-}\left(x^{\prime}\right) \cap V\left(Q_{i_{k}}\right) \neq \emptyset$. Since $\left\{H_{1}, H_{2}, \cdots, H_{c}\right\}$ is the set of maximal symmetrically connected subdigraphs of $D$, there must be an $s^{\prime}$ with $1 \leq s^{\prime} \leq m$ such that $x^{\prime} \in V\left(Q_{i_{h}}\right) \subseteq V\left(H_{i_{s^{\prime}}}\right)$; and there must be an $s^{\prime \prime}$ with $m+1 \leq s^{\prime \prime} \leq m+s$ such that $V\left(Q_{i_{k}}\right) \subseteq V\left(H_{i_{s^{\prime \prime}}}\right)$. Therefore, by Definition 3.4.3(i), $D$ must be partially symmetric. By Theorem 3.4.6, $D$ is supereulerian.

Observe that in Example 3.4.4 (iv), $J_{2}$ is "nearly symmetrically connected". The next examples 3.4.8 and 3.4.10, presents an infinite family of non supereulerian digraphs, such that for each digraph $D$ in the family, every arc of $D$ lies in a subdigraph isomorphic to $J_{2}$. This, in some sense, indicates that Corollary 3.4.7 is best possible. 
Example 3.4.8. Let $\alpha, \beta, k>0$ be integers with $\alpha, \beta \geq k+1$, and let $A$ and $B$ be two disjoint set of vertices with $|A|=\alpha$ and $|B|=\beta$. Let $\ell \geq \alpha \beta+1$ be an integer, and $U$ be a set of vertices disjoint from $A \cup B$ with $|U|=2 \ell$. We construct a digraph $J=J(\alpha, \beta, k, \ell)$ such that $V(J)=A \cup B \cup U$ and the arcs of $J$ are given as required in (J1) and (J2) below.

(J1) $D[A \cup B] \cong K_{\alpha+\beta}^{*}$ is a complete digraph.

(J2) Write $U=\left\{u_{1}, u_{1}^{\prime}, u_{2}, u_{2}^{\prime}, \cdots, u_{\ell}, u_{\ell}^{\prime}\right\}$ such that for each $1 \leq i \leq \ell, J\left[\left\{u_{i}, u_{i}^{\prime}\right\}\right] \cong K_{2}^{*}$, $N_{J}^{+}\left(u_{i}\right)=N_{J}^{+}\left(u_{i}^{\prime}\right)=A$ and $N_{J}^{-}\left(u_{i}\right)=N_{J}^{-}\left(u_{i}^{\prime}\right)=B$. (See Figure 3)

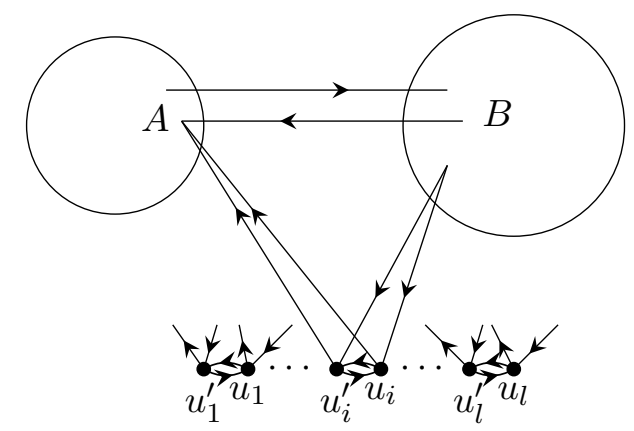

Figure 3. The digraph $D=D(\alpha, \beta, k, \ell)$.

Proposition 3.4.9. Let $J=J(\alpha, \beta, k, \ell)$ for some given parameters $\alpha, \beta, k$ and $\ell$ as defined in Example 3.4.8. Then each of the following holds.

(i) $\lambda(J)>k$.

(ii) Every arc of $J$ lies in a subdigraph isomorphic to $J_{2}$.

(iii) $D$ is not supereulerian.

Proof. (i) The proof (i) similar to that of Proposition 3.2.3(i), and will be omitted.

(ii) Let $a=(u, v)$ be an $\operatorname{arc}$ in $D$. If $u, v \in A \cup B$, then by Example 3.4.8(D2), $a$ is in $K_{\alpha+\beta}^{*}$ and so as a lies in a subdigraph isomorphic to $J_{2}$. Thus we may assume that either $a \in A\left(D\left[\left\{u_{i}, u_{i}^{\prime}\right\}\right]\right)$ for some $i$, or $a \in(B, U)_{D} \cup(U, A)_{D}$. In any case, by Example 3.4.8(J2), for any $w \in A$ and $w^{\prime} \in B$, and for any $i$ with $1 \leq i \leq \ell, J\left[\left\{w, w^{\prime}, u_{i}, u_{i}^{\prime}\right\}\right] \cong J_{2}$. Hence (ii) must hold.

(iii) We apply Proposition 3.2.1. By (J1), $J[A \cup B] \cong K_{\alpha+\beta}^{*}$, and so $\left|\partial_{J}^{+}(A)\right|=\alpha \beta$. By (J2), $\left|(U, B)_{J}\right|=0$ and so $\tau(U)=|U|>\alpha \beta$. It follows that

$$
h(U, A)=\left|\partial_{J}^{+}(A)\right|+\left|(U, B)_{J}\right|-\tau(U)=\alpha \beta-|U|<0
$$

It follows from Proposition 3.2.1 that $J$ is not supereulerian. 
Example 3.4.10. (1) Let $H_{1}$ and $H_{2}$ be digraph with $V\left(H_{1}\right)=V\left(H_{2}\right)=\left\{v_{1}, v_{2}, v_{3}, v_{4}\right\}$ and $A\left(H_{1}\right)=\left\{\left(v_{1}, v_{2}\right),\left(v_{2}, v_{1}\right),\left(v_{1}, v_{4}\right),\left(v_{2}, v_{3}\right),\left(v_{3}, v_{1}\right),\left(v_{4}, v_{3}\right),\left(v_{3}, v_{4}\right),\left(v_{2}, v_{4}\right)\right\}$, and $A\left(H_{2}\right)=$ $\left\{\left(v_{1}, v_{3}\right),\left(v_{1}, v_{4}\right),\left(v_{4}, v_{3}\right)\right.$, $\left.\left(v_{3}, v_{4}\right),\left(v_{3}, v_{2}\right),\left(v_{2}, v_{4}\right),\left(v_{2}, v_{1}\right),\left(v_{1}, v_{2}\right)\right\}$. Then we have these observations.

(i) For $i \in\{1,2\}, H_{i}$ are strong.

(ii) For $i \in\{1,2\}, H_{i}$ is not partially symmetric digraphs, as depicted in Figure 4.

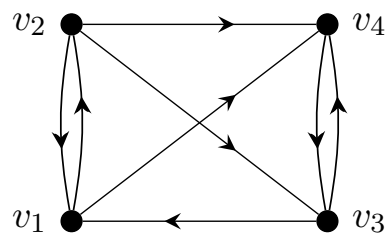

$H_{1}$

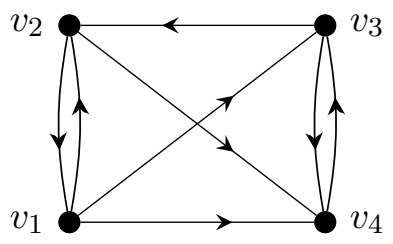

$H_{2}$

Figure 4: $H_{1}$ and $H_{2}$.

(2) We will present $\mathrm{H}_{1} \oplus_{2} \mathrm{H}_{2}$ digraph, and then we will use the $\mathrm{H}_{1} \oplus_{2} \mathrm{H}_{2}$ digraph to built an infinite family of non supereulerian digraphs, such that for each digraph $D$ in the family, every arc of $D$ lies in a subdigraph isomorphic to $H_{i}$ for $i=1,2$.

(i) Let $V\left(H_{1} \oplus_{2} H_{2}\right)=\left\{v_{1}, v_{2}, v_{3}, v_{4}, v_{5}, v_{6}\right\}$ and $A\left(H_{1} \oplus_{2} H_{2}\right)=\left\{\left(v_{1}, v_{2}\right),\left(v_{2}, v_{1}\right),\left(v_{1}, v_{4}\right),\left(v_{2}, v_{3}\right)\right.$, $\left(v_{2}, v_{4}\right),\left(v_{3}, v_{1}\right)$,

$\left.\left(v_{4}, v_{3}\right),\left(v_{3}, v_{4}\right),\left(v_{3}, v_{6}\right),\left(v_{3}, v_{5}\right),\left(v_{4}, v_{5}\right),\left(v_{5}, v_{6}\right),\left(v_{6}, v_{5}\right),\left(v_{6}, v_{4}\right)\right\}$. Then we observe $H_{1} \oplus_{2} H_{2}$ may not be supereulerian since $d_{H_{1} \oplus_{2} H_{2}}^{+}\left(v_{4}\right)=1$ as depicted in Figure 5

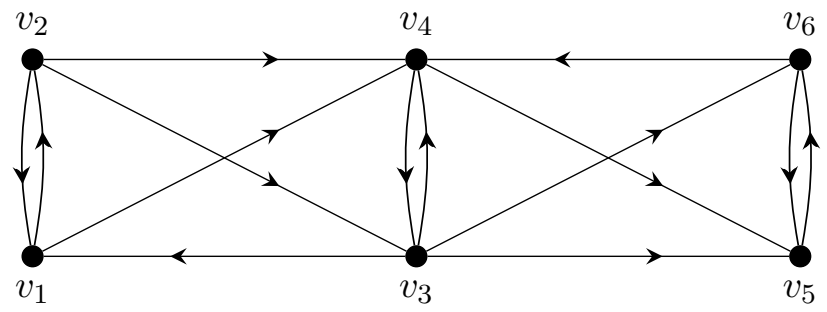

Figure 5: $\mathrm{H}_{1} \oplus_{2} \mathrm{H}_{2}$.

(ii) Using the notation in Figure 5, we can fix the vertex set of one of the two $K_{2}^{*}$ 's in $H_{1} \oplus_{2} H_{2}, H_{1} \oplus_{2} H_{2}\left[\left\{v_{1}, v_{2}\right\}\right]$ and $H_{1} \oplus_{2} H_{2}\left[\left\{v_{5}, v_{6}\right\}\right]$, and define it as a distinguished pair. Let $\{x, y\}$ be a distinguished pair of $H_{1} \oplus_{2} H_{2}$, and denote $H_{1} \oplus_{2} H_{2}$ by $D(x, y):=H_{1} \oplus_{2} H_{2}(x, y)$. 
Let $D_{1}\left(x^{\prime}, y^{\prime}\right)$ and $D_{2}\left(x^{\prime \prime}, y^{\prime \prime}\right)$ be the two copies of $D\left(v_{1}, v_{2}\right)$. Obtain a new digraph $D_{1} \oplus_{2} D_{2}$ from $D_{1}$ and $D_{2}$ by identifying $x^{\prime}$ and $x^{\prime \prime}$ to form a new vertex $x$, and identifying $y^{\prime}$ and $y^{\prime \prime}$ to form a new vertex $y$. See figure 6 for an drawing of $D_{1} \oplus_{2} D_{2}$ with $x=v_{1}$ and $y=v_{2}$.

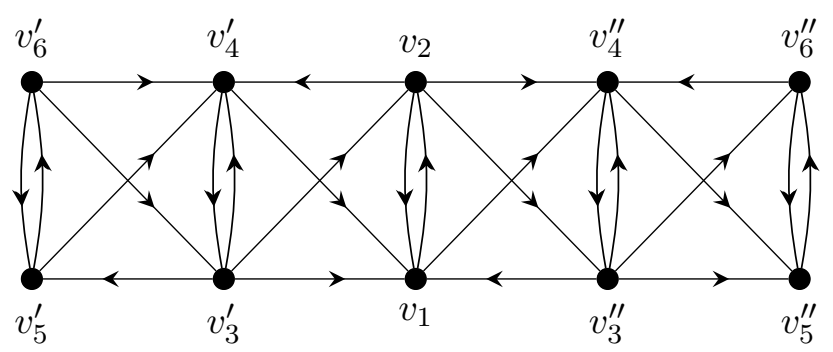

Figure 6: $D_{1} \oplus_{2} D_{2}$.

\subsection{Locally $k$-Eulerian Digraphs}

Let $D$ be a weakly connected digraph and $k \geq 2$ be an integer. We call $D$ a locally $k$-Eulerian digraph if for any arc $a \in A(D)$, there exists an Eulerian subdigraph $H_{a}$ contain $a$ in $D$ contains $a$, such that $\left|A\left(H_{a}\right)\right| \leq k$.

Proposition 3.5.1. Let $\mathcal{E}_{k}$ be the family of all locally $k$-Eulerian digraphs and let $D$ be a weakly connected locally $\mathcal{E}_{k}$ digraph. Each of the following holds.

(I) $D$ is a strong.

(II) There exist an Example of infinite family of digraphs show that for any integer $k \geq 3$, there exists locally $k$-Eulerian non supereulerian digraph $D$ with $\lambda(D) \geq k$.

(III)If for any $H \in \mathcal{E}_{k}, H$ is symmetrically connected, then $D$ is supereulerian.

Proof. (I) Assume that $D$ is not strongly connected. Then there exists a proper nonempty subset $\emptyset \neq X \subset V(D)$ with $\left|\partial_{D}^{+}(X)\right|=0$. By assumption $D$ is weakly connected, $D$ contains an $\operatorname{arc} a=(u, v) \in(V(D)-X), X)_{D}$. Since $D$ is a locally $\mathcal{E}_{k}$ digraph, there exist an Eulerian subdigraph $H_{a}$ contains $a \in A\left(H_{a}\right)$. Since $\left.(u, v) \in(V(D)-X), X\right)_{D}$, we conclude that $\left.\emptyset \neq A\left(H_{a}\right) \cap(X, V(D)-X)\right)_{D} \subseteq \partial_{D}^{+}(X)$, contrary to the assumption $\left|\partial_{D}^{+}(X)\right|=0$. This proves that $D$ must be strong.

Example 3.5.2. Let $h, l \geq 1$ be integers and let $A$ and $B$ be two disjoint set of vertices with 
$|A|=h$ and $|B|=l$. Let $U$ be a set of vertices disjoint from $A \cup B$ with $|U|=h \times l+1$. We construct a digraph $E=E(h, l, h \times l+1)$ such that $V(E)=A \cup B \cup U$ and the arcs of $E$ are given as required in (E1) and (E2) below.

(E1) $D[A \cup B] \cong K_{h+l}^{*}$ is a complete digraph.

(E2) For every vertex $u \in U$, and for every $v \in A,(u, v) \in A(D)$ and for every $w \in B$, $(w, u) \in A(D)$. Thus for any $u \in U$, we have $N_{D}^{+}(u)=A$ and $N_{D}^{-}(u)=B$. No two vertices in $U$ are adjacent.

Proposition 3.5.3. Let $E=E(h, l, h \times l+1)$ for some given parameters $h, l$ as defined in Example 3.5.2. Then each of the following holds.

(i) $\lambda(D) \geq \min \{h, l\}$.

(ii) For any arc $a \in A(D)$, there exists a subdigraph $H_{a}$ of $D$ contain a such that $\left|A\left(H_{a}\right)\right| \leq$ $h+l+1$.

(iii) $D$ is not supereulerian.

Proof. (i) The proof (i) similar to that of Proposition 3.2.3(i), and will be omitted.

(ii) Now let $a=(x, y)$ be an arc in $A(D)$. If $a \in A(D[A \cup B])$, then by (D1), $D[A \cup B] \cong$ $K_{h+l}^{*}$. By our assumption there exist many of subdigraphs say $H_{a}$ contain $a$ with each of them $\left|A\left(H_{a}\right)\right| \leq h+l$, implies the condition on subdigraphs is hold. Hence by (D2), we may assume that $(x, y) \in(A \cup B, U)_{D}$ or $(x, y) \in(U, A \cup B)_{D}$. In either case, let $A^{\prime} \subseteq A$ and $B^{\prime} \subseteq B$ be subsets with $\left|A^{\prime}\right|=\left|N_{H}^{+}(v)\right|$ and $\left|B^{\prime}\right|=\left|N_{H}^{-}(v)\right|$, respectively. Then $D\left[A^{\prime} \cup B^{\prime} \cup\{x, y\}\right]$ contain a an Eulerian subdigraph $H_{a}$ contains $a$ with $\left|A\left(H_{a}\right)\right| \leq h+l+1$, then our condition is hold. This justifies that $D$ is a locally k-Eulerian digraph.

(iii) We apply Proposition 3.2.1. By (E1), $E[A \cup B] \cong K_{h+l}^{*}$, and so $\left|\partial_{E}^{+}(A)\right|=h \times l$. By (E2), $\left|(U, B)_{E}\right|=0$ and so $\tau(U)=|U|=h \times l+1$. It follows that

$$
h(U, A)=\left|\partial_{E}^{+}(A)\right|+\left|(U, B)_{E}\right|-\tau(U)=h \times l-|U|<0 .
$$

It follows from Proposition 3.2.1 that $E$ is not supereulerian.

(III) If for any $H \in \mathcal{E}_{k}, H$ is symmetrically connected, then by Corollary $3.3 .3, D$ is supereulerian. 


\subsection{A k-free trailable Digraphs}

Definition 3.6.1. Let $D$ a digraph and $k \geq 2$ be an integer, then $D$ called a $k$-free trailable digraph, if for any $X \subseteq V(D)$ with $|X|=k$. Then $D[X]$ the digraph induced by $X$ has spanning closed ditrail. For simplicity of condition, if every $k$ vertices lies in closed ditrail of $D$.

We will begin with this section by introducing the following worths theorems in supereulerian digraphs:

Theorem 3.6.2. (Jensen and Maddalini, Theorem(3.7) [17])

Let $D$ be a strong simple digraph on $n$ vertices. If $d(x)+d(y) \geq 2 n-3$ for any pair of non adjacent vertices $x$ and $y$, then $D$ is supereulerian.

Theorem 3.6.3. (Hong, Lai and Liu, Theorem(3.4) of [34])

Let $D$ be a strong digraph with $|V(D)|=n$ and $\delta^{+}(D) \geq 4$ and $\delta^{-}(D) \geq 4$; If $\delta^{+}(D)+$ $\delta^{-}(D) \geq n-4$ then $D$ is supereulerian.

Theorem 3.6.4. Let $k \geq 2$ be an integer number. Let $D$ be a nonsingular digraph with

$$
|V(D)|=n \geq\left\{\begin{aligned}
2 k-3 & \text { if } 2 \leq k \leq 6 \\
k+3 & \text { if } 6 \leq k \leq 9 \\
2 k-6 & \text { if } 9 \leq k
\end{aligned}\right.
$$

or $n=k$. If $\forall X \subseteq V(D)$ with $|X|=k, D[X]$ has spanning closed ditrail, then $D$ is supereulerian.

Proof. It is clear that $D$ is strong because for any $x, y \in V(D)$ by our condition there exists a $C_{k}$ contains $x$ and $y$, so there exist a dipath from $x$ to $y$ and a dipath from $y$ to $x$. If $n=k$, then $D$ is a Hamiltonian and we are done. If $n>k$, then let $X_{0} \subset V(D)$ with $\left|X_{0}\right|=k$ and $v \in X_{0}$. Then by our condition $v$ has an out-neighbor $y_{1}$ in $X_{0}$. Let $\left\{x_{1}, x_{2}, \ldots, x_{n-k}\right\}=V(D)-X_{0}$ and $X_{1}=\left(X_{0} \cup\left\{x_{1}\right\}\right)-y_{1}$. Then $v$ has a nother out-neighbor $y_{2}$ in $X_{1}$. Repeating this procedure $v$ has at least $n-k+1$ out-neighbors in $D$. By the same procedure $v$ has at least $n-k+1$ in-neighbors. Therefore, $d(v) \geq 2(n-k+1)$.

(I) By using Theorem 3.6.2, we determine when $d(x)+d(y) \geq 2 n-3 \forall x, y \in V(D)$ 


$$
\begin{gathered}
\Rightarrow 4(n-k+1) \geq 2 n-3 \\
\Rightarrow 4 n-4 k+4 \geq 2 n-3 \\
\Rightarrow 2 n \geq 4 k-7 \\
\Rightarrow n \geq 2 k-\frac{7}{2} \\
n \geq 2 k-3
\end{gathered}
$$

It means that:

When $n \geq 2 k-3$, then $D$ is supereulerian.

(II) Since $v$ has at least $n-k+1$ in-neighbors in $D$ and has at least $n-k+1$ out-neighbors in $D$ as well. Then

(A) By using the first condition of Theorem 3.6.3 that $\delta^{+}(D) \geq 4$ and $\delta^{-}(D) \geq 4$ we have the following:

$$
\begin{aligned}
& n-k+1 \geq 4 \\
& \Rightarrow n \geq k+3 .
\end{aligned}
$$

(B) By using the second condition of Theorem 3.6.3:

$$
\begin{gathered}
\delta^{+}(D)+\delta^{-}(D) \geq n-4 \\
\Rightarrow 2(n-k+1) \geq n-4 \\
\Rightarrow n \geq 2 k-6 .
\end{gathered}
$$

Combining (A) with (B) implies that when $n \geq \max \{2 k-6, k+3\}$, then $D$ is supereulerian.

Combining (I) with (II), we get that when $n \geq \min \{\max \{2 k-5, k+3\}, 2 k-3\}$, then $D$ is supereulerian. Thus, if

$$
n \geq\left\{\begin{aligned}
2 k-3 & \text { if } 2 \leq k \leq 6 \\
k+3 & \text { if } 6 \leq k \leq 9 \\
2 k-6 & \text { if } 9 \leq k
\end{aligned}\right.
$$

then $D$ is supereulerian.

In the following Definition, is a special case of the general case of Definition 3.6.1: 
Definition 3.6.5. Let $D$ a digraph and $k \geq 2$ be an integer, then $D$ called a $k$-free trailable digraph, if for any $X \subseteq V(D)$ with $|X|=k$. Then $D[X]$ the digraph induced by $X$ has a dicycle $C_{k}$ with $k$ vertices. For simplicity of condition, if every $k$ vertices lies in closed dicycle $C_{k}$ of $D$.

Theorem 3.6.6. Let $k \geq 2$ be an integer number. Let $D$ be a nonsingular digraph with

$$
|V(D)|=n \geq\left\{\begin{aligned}
2 k-3 & \text { if } 2 \leq k \leq 6 \\
k+3 & \text { if } 6 \leq k \leq 9 \\
2 k-6 & \text { if } 9 \leq k \leq 13 \\
k+\left\lceil\frac{k}{2}\right\rceil & \text { if } 14 \leq k
\end{aligned}\right.
$$

or $n=k$. If for any $X \subseteq V(D)$ with $|X|=k, D[X]$ has a dicycle $C_{k}$, then $D$ is supereulerian.

Proof. It is clear that $D$ is strong because for any $x, y \in V(D)$ by our condition there exists a $C_{k}$ contains $x$ and $y$, so there exist a dipath from $x$ to $y$ and a dipath from $y$ to $x$. If $n=k$, then $D$ is a Hamiltonian and we are done. For $n>k$, by Lemma 2.2.14 we have that $d(v) \geq 2(n-k+1)$ for all vertex $v \in V(D)$.

(I) By using Theorem 3.6.2, we determine when $d(x)+d(y) \geq 2 n-3 \forall x, y \in V(D)$. Then we have that $4(n-k+1) \geq 2 n-3$ implies that $n \geq 2 k-3$. That mean when $n \geq 2 k-3$ then $D$ is supereulerian.

(II) Since every vertex $v \in V(D)$ has at least $n-k+1$ in-neighbors and at least $n-k+1$ out-neighbors . We have the following:

(A) By using the first condition of Theorem 3.6.3, we determine when $\delta^{+}(D) \geq 4$ and $\delta^{-}(D) \geq$ 4 , implies that $n-k+1 \geq 4$. We have that $n \geq k+3$.

(B) By using the second condition of Theorem 3.6.3, we determine when $\delta^{+}(D)+\delta^{-}(D) \geq n-3$. Then we have that $2(n-k+1) \geq n-3$ that give us $n \geq 2 k-5$

Then, by using (A) and (B) together we get that when $n \geq \max \{2 k-5, k+3\}$, then $D$ is supereulerian.

Hence, by combining (I) and (II), we get that when $n \geq \min \{\max \{2 k-5, k+3\}, 2 k-3\}$, then $D$ is supereulerian. Thus, if

$$
n \geq\left\{\begin{aligned}
2 k-3 & \text { if } 2 \leq k \leq 6 \\
k+3 & \text { if } 6 \leq k \leq 9 \\
2 k-6 & \text { if } 9 \leq k \leq 13
\end{aligned}\right.
$$


then $D$ is supereulerian.

Now we need to prove the reminder case when $n \geq k+\left\lceil\frac{k}{2}\right\rceil$. Let $X \subset V(D)$ with $|X|=k$. By definition of $D$ let $C_{k}$ be a dicycle consist of all vertices of $X$. Let $Y=V(D)-V\left(C_{k}\right)=\left\lceil\frac{k}{2}\right\rceil$ . Let $\bar{X} \subset V\left(C_{k}\right)$ consist of nonadjacent vertices of $C_{k}$. Then we have the following cases:

- Case(1) If $14 \leq k$, such that $k$ is an even number.

By picking $\bar{X}=\left\lceil\frac{k}{2}\right\rceil=\frac{k}{2}$ and join them with $Y=\left\lceil\frac{k}{2}\right\rceil=\frac{k}{2}$, we get that $\bar{X} \cup Y=\frac{k}{2}+\frac{k}{2}=k$. Since $|\bar{X} \cup Y|=k$ then by definition of $D$ consider a cycle $\bar{C}_{k}$ consist of $\bar{X} \cup Y$. Let $v \in \bar{X}$, then start at the vertex $v$ and go over all vertices belong to $\bar{C}_{k}$ until back the same starting vertex, then move to all vertices belong to $C_{k}$ until back to same vertex. Hence, we prove that $D$ is supereulerian.

- Case(2) If $14 \leq k$, such that $k$ is an odd number.

By picking $\bar{X}=\left\lceil\frac{k}{2}\right\rceil=\frac{k}{2}$ and join them with $Y=\left\lceil\frac{k}{2}\right\rceil=\left(\frac{k}{2}\right)+1$, we get that $\bar{X} \cup Y=$ $\frac{k}{2}+\left\lceil\frac{k}{2}\right\rceil=\left(\frac{k}{2}+\frac{k}{2}\right)+1=(k-1)+1=k$. Since $|\bar{X} \cup Y|=k$ then by definition of $D$ consider a cycle $\bar{C}_{k}$ consist of $\bar{X} \cup Y$. Let $v \in \bar{X}$, then start at the vertex $v$ and go over all vertices belong to $\bar{C}_{k}$ until back the same starting vertex, then move to all vertices belong to $C_{k}$ until back to same vertex. Hence, we prove that $D$ is supereulerian.

We conclude from case(1) and (2) that $D$ is supereulerian. 


\section{Chapter 4}

\section{On a class of supereulerian digraphs}

\subsection{Introduction}

We consider finite graphs and digraphs, and undefined terms and notations will follow [1] for graphs and [2] for digraphs. Throughout this paper, the notation $(u, v)$ denotes an arc oriented from $u$ to $v$. A digraph $D$ is strict if it contains no parallel arcs nor loops; and is symmetric if for any vertices $u, v \in V(D)$, if $(u, v) \in A(D)$, then $(v, u) \in A(D)$. If two $\operatorname{arcs}$ of $D$ have a common vertex, we say that these two arcs are adjacent in $D$. A directed path in a digraph $D$ from a vertex $u$ to a vertex $v$ is called a $(u, v)$-dipath. To emphasize the distinction between graphs and digraphs, a directed cycle or path in a digraph is often referred as a dicycle or dipath. A dipath $P$ is a hamiltonian dipath if $V(P)=V(D)$. A digraph $D$ is hamiltonian if $D$ contains a hamiltonian dicycle. An $(x, y)$-hamiltonian dipath is a hamiltonian dipath from $x$ to $y$. A digraph $D$ is hamiltonian-connected if $D$ has an $(x, y)$-hamiltonian dipath for every choice of distinct vertices $x, y \in V(D)$.

As in $[2], \lambda(D)$ denotes the arc-strong-connectivity of $D$. A digraph $D$ is strong if and only if $\lambda(D) \geq 1$. For $X, Y \subseteq V(D)$, we define

$$
(X, Y)_{D}=\{(x, y) \in A(D): x \in X \text { and } y \in Y\} ; \text { and } \partial_{D}^{+}(X)=(X, V(D)-X)_{D}
$$

For a subset $A^{\prime} \subseteq A(D)$ the subdigraph arc-induced by $A^{\prime}$ is the digraph $D\left[A^{\prime}\right]=\left(V^{\prime}, A^{\prime}\right)$, where $V^{\prime}$ is the set of vertices in $V$ which are incident with at least one arc in $A^{\prime}$.

Let

$$
d_{D}^{+}(X)=\left|\partial_{D}^{+}(X)\right|, \text { and } d_{D}^{-}(X)=\left|\partial_{D}^{-}(X)\right|
$$


When $X=\{v\}$, we write $d_{D}^{+}(v)=\left|\partial_{D}^{+}\{v\}\right|$ and $d_{D}^{-}(v)=\left|\partial_{D}^{-}\{v\}\right|$. Let $N_{D}^{+}(v)=\{u \in V(D)-v$ : $(v, u) \in A(D)\}$ and $N_{D}^{-}(v)=\{u \in V(D)-v:(u, v) \in A(D)\}$ denote the out-neighbourhood and in-neighbourhood of $v$ in $D$, respectively. Vertices in $N_{D}^{+}(v), N_{D}^{-}(v)$ are called the outneighbours, in-neighbours of $v$.

Boesch, Suffel, and Tindell [30] in 1977 proposed the supereulerian problem, which seeks to characterize graphs that have spanning eulerian subgraphs. They indicated that this problem would be very difficult. Pulleyblank [26] later in 1979 proved that determining whether a graph is supereulerian, even within planar graphs, is NP-complete. Catlin [20] in 1992 presented the first survey on supereulerian graphs. Chen et al [21] surveyed the reduction method associated with the supereulerian problem and their applications. An updated survey presenting the more recent developments can be found in [35].

It is natural to consider the supereulerian problem in digraphs. A digraph $D$ is eulerian if it contains a closed ditrail $W$ such that $A(W)=A(D)$, or, equivalently, if $D$ is strong and for any $v \in V(D), d_{D}^{+}(v)=d_{D}^{-}(v)$. A digraph $D$ is supereulerian if $D$ contains a closed ditrail $W$ such that $V(W)=V(D)$, or, equivalently, if $D$ contains a spanning eulerian subdigraph. Some recent developments on supereulerian digraphs are given in $[17,27,31,32,34]$.

A central problem is to determine or characterize supereulerian digraphs. In Section 2, the 2-sum $D_{1} \oplus_{2} D_{2}$ of two digraphs $D_{1}$ and $D_{2}$ is defined, and some basic properties of 2-sums are discussed. We will observe that a 2-sum of two supereulerian (or even hamiltonian) digraphs may not be supereulerian. Thus it is natural to seek sufficient conditions on $D_{1}$ and $D_{2}$ for the 2-sum of $D_{1}$ and $D_{2}$ to be supereulerian. In the last section, we will present several sufficient conditions for supereulerian 2-sums of digraphs. In particular, we show that if $D_{1}$ and $D_{2}$ are either symmetrically connected or partially symmetric (to be defined in Section 3), then $D_{1} \oplus_{2} D_{2}$ is supereulerian.

\subsection{The 2-sums of digraphs}

The definition and some elementary properties of the 2-sums of digraphs are presented in this section. A digraph is nontrivial if it contains at least one arc. Throughout this section, all digraphs are assumed to be nontrivial.

Definition 4.2.1. Let $D_{1}$ and $D_{2}$ be two vertex disjoint digraphs, and let $a_{1}=\left(v_{11}, v_{12}\right) \in A\left(D_{1}\right)$ and $a_{2}=\left(v_{21}, v_{22}\right) \in A\left(D_{2}\right)$ be two distinguished arcs. The 2-sum $D_{1} \oplus_{a_{1}, a_{2}} D_{2}$ of $D_{1}$ and $D_{2}$ with base arcs $a_{1}$ and $a_{2}$ is obtained from the union of $D_{1}$ and $D_{2}-a_{2}$ by identifying $v_{11}$ with 
$v_{21}$ and $v_{12}$ with $v_{22}$, respectively. When the arcs $a_{1}$ and $a_{2}$ are not emphasized or is understood from the context, we often use $D_{1} \oplus_{2} D_{2}$ for $D_{1} \oplus_{a_{1}, a_{2}} D_{2}$.

Lemma 4.2.2. Let $D_{1}$ and $D_{2}$ be two vertex disjoint strong digraphs. Then

$$
\lambda\left(D_{1} \oplus_{2} D_{2}\right) \geq \min \left\{\lambda\left(D_{1}\right), \lambda\left(D_{2}\right)\right\}
$$

Proof. Let $k \geq 0$ be an integer such that $\min \left\{\lambda\left(D_{1}\right), \lambda\left(D_{2}\right)\right\}=k$, and let $\lambda\left(D_{1} \oplus_{2} D_{2}\right)=k^{\prime}$. We shall show that $k^{\prime} \geq k$. By (1.1), there exists a proper nonempty vertex subset $X \subset V\left(D_{1} \oplus_{2} D_{2}\right)$ such that $\left|\partial_{D_{1} \oplus_{2} D_{2}}^{+}(X)\right|=k^{\prime}$. Let $S=\partial_{D_{1} \oplus_{2} D_{2}}^{+}(X)$. We argue by contradiction and assume that $k^{\prime}<k$.

By Definition 4.2.1, we have $v_{11}=v_{21} \in V\left(D_{2}\right)$ and $v_{12}=v_{22} \in V\left(D_{2}\right)$ in $D_{1} \oplus_{2} D_{2}$. If $X \cap V\left(D_{1}\right) \neq \emptyset$ and $X \cap V\left(D_{2}\right)=\emptyset$, we obtain that $v_{11}=v_{21} \notin X$ and $v_{12}=v_{22} \notin X$, then $X \subset V\left(D_{1}\right)$ and $S=\partial_{D_{1}}^{+}(X)$. It follows by (1.1) that $k^{\prime}=|S| \geq \lambda\left(D_{1}\right) \geq k$, contrary to the assumption that $k^{\prime}<k$. Similarly, if $X \cap V\left(D_{1}\right)=\emptyset$ and $X \cap V\left(D_{2}\right) \neq \emptyset$, then $X \subset V\left(D_{2}\right)$ and $S=\partial_{D_{2}}^{+}(X)$, hence a contradiction to the assumption that $k^{\prime}<k$ is obtained from $k^{\prime}=|S| \geq \lambda\left(D_{2}\right) \geq k$.

Thus we may assume that $X \cap V\left(D_{1}\right) \neq \emptyset$ and $X \cap V\left(D_{2}\right) \neq \emptyset$. Let $X^{\prime}=X \cap V\left(D_{1}\right)$. Then $X^{\prime}$ is a proper nonempty subset of $V\left(D_{1}\right)$, and $\partial_{D_{1}}^{+}\left(X^{\prime}\right) \subseteq S$. It follows by (1.1) that $k^{\prime}=|S| \geq\left|\partial_{D_{1}}^{+}\left(X^{\prime}\right)\right| \geq \lambda\left(D_{1}\right) \geq k$ contrary to the assumption that $k^{\prime}<k$.

Example 4.2.3. The converse of Lemma 4.2.2 may not always stand, as indicated by the example below, depicted in Figure 1. Let $V\left(D_{1}\right)=\left\{v_{11}, v_{12}, v_{13}, v_{14}\right\}$ and $V\left(D_{2}\right)=\left\{v_{21}, v_{22}, v_{23}, v_{24}\right\}$. Let $A\left(D_{1}\right)=\left\{\left(v_{11}, v_{12}\right),\left(v_{13}, v_{12}\right),\left(v_{14}, v_{13}\right),\left(v_{11}, v_{14}\right),\left(v_{11}, v_{13}\right),\left(v_{14}, v_{12}\right)\right\}$ and $A\left(D_{2}\right)=\left\{\left(v_{21}, v_{22}\right)\right.$, $\left.\left(v_{22}, v_{23}\right),\left(v_{23}, v_{24}\right),\left(v_{24}, v_{21}\right),\left(v_{23}, v_{21}\right),\left(v_{24}, v_{22}\right)\right\}$. Let $a_{1}=\left(v_{11}, v_{12}\right)$ and $a_{2}=\left(v_{21}, v_{22}\right)$. Then it is routine to verify that $\lambda\left(D_{1} \oplus_{a_{1}, a_{2}} D_{2}\right) \geq 1$. While $D_{2}$ is strong, the digraph $D_{1}$ contains a vertex $v_{11}$ with $d_{D_{1}}^{-}\left(v_{11}\right)=0$, and so $\lambda\left(D_{1}\right)=0$.

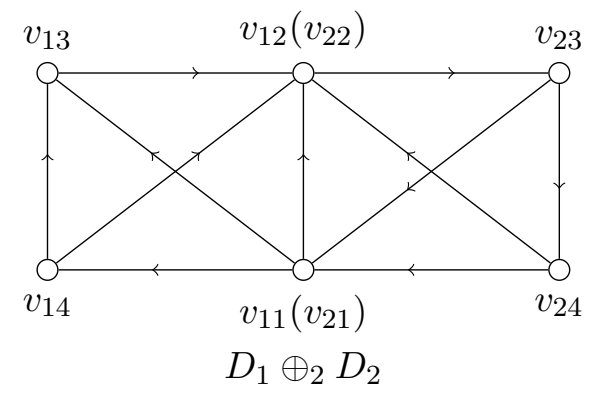

Figure 1. $\lambda\left(D_{1} \oplus_{2} D_{2}\right)=1$ but $\min \left\{\lambda\left(D_{1}\right), \lambda\left(D_{2}\right)\right\}=0$. 
Lemma 4.2.4. A digraph $D$ is not supereulerian if for some integer $m>0, V(D)$ has vertex disjoint subsets $\left\{B, B_{1}, \ldots, B_{m}\right\}$ satisfying both of the following:

(i) $N_{D}^{-}\left(B_{i}\right) \subseteq B, \forall i \in\{1,2, \ldots, m\}$.

(ii) $\left|\partial_{D}^{-}(B)\right| \leq m-1$.

Proof. By contradiction, we assume that both (i) and (ii) hold and $D$ is supereulerian. Let $S$ be a spanning eulerian subdigraph of $D$, then $B \subset V(S)=V(D)$ and $A(S) \subset A(D)$. Since $S$ is eulerian, for any subset $X \subset V(S)$, it follows that $\left|\partial_{S}^{+}(X)\right|=\left|\partial_{S}^{-}(X)\right|$. Thus, by (ii), we conclude that

$$
\left|\partial_{D}^{+}(B) \cap A(S)\right|=\left|\partial_{D}^{-}(B) \cap A(S)\right| \leq\left|\partial_{D}^{-}(B)\right| \leq m-1
$$

By (i) and by (4.1), there must be a $B_{j}$ with $j \in\{1,2, \ldots, m\}$ such that $\partial_{D}^{-}\left(B_{j}\right) \cap A(S)=\emptyset$, contrary to the assumption that $V(S)=V(D)$.

Lemma 4.2.4 can be applied to find examples of hamiltonian digraphs whose 2-sum is not supereulerian, as shown in Example 4.2.5 below.

Example 4.2.5. Let $n_{1}, n_{2} \geq 3$ be integers and $C_{n_{1}}$ and $C_{n_{2}}$ be two vertex disjoint dicycles with length $n_{1}$ and $n_{2}$, respectively. We claim that $C_{n_{1}} \oplus_{2} C_{n_{2}}$ is not supereulerian. To justify this claim, we denote $V\left(C_{n_{1}}\right)=\left\{v_{11}, v_{12}, \ldots, v_{1 n_{1}}\right\}$, and $V\left(C_{n_{2}}\right)=\left\{v_{21}, v_{22}, \ldots, v_{2 n_{2}}\right\}$. Without loss of generality, we assume that $a_{1}=\left(v_{11}, v_{12}\right)$ and $a_{2}=\left(v_{21}, v_{22}\right)$, and $C_{n_{1}} \oplus_{2} C_{n_{2}}=C_{n_{1}} \oplus_{a_{1}, a_{2}} C_{n_{2}}$. Let $B, B_{1}$ and $B_{2}$ be subdigraphs of $C_{n_{1}} \oplus_{2} C_{n_{2}}$ with $V(B)=\left\{v_{12}\right\}, V\left(B_{1}\right)=\left\{v_{13}\right\}$ and $V\left(B_{2}\right)=$ $\left\{v_{23}\right\}$, respectively. By Lemma 4.2.4, we conclude that $C_{n_{1}} \oplus_{2} C_{n_{2}}$ is not supereulerian (See Figure 2).

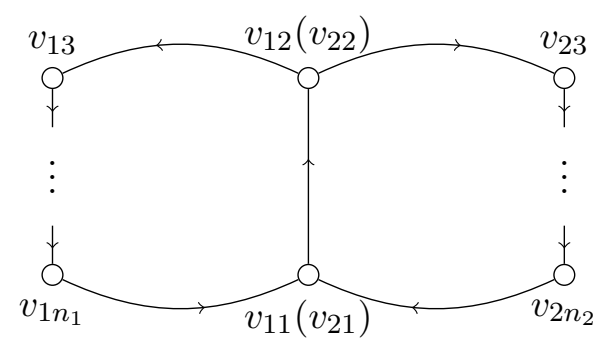

Figure 2. The 2-sum $C_{n_{1}} \oplus_{2} C_{n_{2}}$ of $C_{n_{1}}$ and $C_{n_{2}}$ 


\subsection{Sufficient conditions for supereulerian 2-sums of digraphs}

In this section, we will show several sufficient conditions on $D_{1}$ and $D_{2}$ to assure that the 2 -sum $D_{1} \oplus_{2} D_{2}$ is supereulerian.

Proposition 4.3.1. Let $D_{1}$ and $D_{2}$ be two vertex disjoint supereulerian digraphs with $a_{1}=$ $\left(v_{11}, v_{12}\right) \in A\left(D_{1}\right)$ and $a_{2}=\left(v_{21}, v_{22}\right) \in A\left(D_{2}\right)$, and let $D_{1} \oplus_{2} D_{2}$ denote $D_{1} \oplus_{a_{1}, a_{2}} D_{2}$. Each of the following holds.

(i) For some $i \in\{1,2\}$, if $D_{i}$ has a spanning eulerian subdigraph $S_{i}$ such that $a_{i} \notin A\left(S_{i}\right)$, then $D_{1} \oplus_{2} D_{2}$ is supereulerian.

(ii) If for some $i \in\{1,2\}, D_{i}$ is hamiltonian-connected, then $D_{1} \oplus_{2} D_{2}$ is supereulerian.

Proof. (i) Since $D_{1}$ and $D_{2}$ are supereulerian digraphs, $D_{1}$ and $D_{2}$ are strongly connected, and so by Lemma 4.2.2, $D_{1} \oplus_{2} D_{2}$ is also strongly connected. Without loss of generality, we assume that $i=1$ and $D_{1}$ has a spanning eulerian subdigraph $S_{1}$ such that $a_{1} \notin A\left(S_{1}\right)$. Since $D_{2}$ is supereulerian, we can pick a spanning eulerian subdigraph $S_{2}^{\prime}$ in $D_{2}$. Then $A\left(S_{1}\right) \cap A\left(S_{2}^{\prime}\right)=\emptyset$ and $V\left(S_{1}\right) \cap V\left(S_{2}^{\prime}\right) \neq \emptyset$. It follows that $D\left[A\left(S_{1}\right) \cup A\left(S_{2}^{\prime}\right)\right]$ is a spanning eulerian subdigraph in $D_{1} \oplus_{2} D_{2}$.

(ii) Without loss of generality, we assume that $i=1$ and $D_{1}$ is hamiltonian-connected, and so $D_{1}$ has a $\left(v_{11}, v_{12}\right)$-hamiltonian dipath $T_{1}$ and a $\left(v_{12}, v_{11}\right)$-hamiltonian dipath $T_{2}$. Since $D_{2}$ is supereulerian, $D_{2}$ contains a spanning eulerian subdigraph $S_{2}^{\prime}$. Define

$$
S=\left\{\begin{array}{ll}
D\left[A\left(T_{1}\right) \cup A\left(S_{2}^{\prime}-\left\{\left(v_{21}, v_{22}\right)\right\}\right)\right] & \text { if }\left(v_{21}, v_{22}\right) \in A\left(S_{2}^{\prime}\right) \\
D\left[\left(A\left(T_{2}\right) \cup\left\{\left(v_{11}, v_{12}\right)\right\}\right) \cup A\left(S_{2}^{\prime}\right)\right] & \text { if }\left(v_{21}, v_{22}\right) \notin A\left(S_{2}^{\prime}\right)
\end{array} .\right.
$$

As in any case, $S$ is strongly connected and every vertex $v \in V(S)$ satisfies $d_{S}^{+}(v)=d_{S}^{-}(v)$, and so $S$ is eulerian. Since $V(S)=V\left(T_{i}\right) \cup V\left(S_{2}^{\prime}\right)=V\left(D_{1}\right) \cup V\left(D_{2}\right)$, for $i \in\{1,2\}$, we conclude that $S$ is a spanning eulerian subdigraph of $D_{1} \oplus_{2} D_{2}$, and so $D_{1} \oplus_{2} D_{2}$ is supereulerian.

If the absence of condition (i) in Proposition 4.3.1 is not sufficient to guarantee the existence $D_{1} \oplus_{2} D_{2}$ be a supereulerian. We have introduce the following counterexample.

Example 4.3.2. (1) Let $H_{1}$ and $H_{2}$ be digraph with $V\left(H_{1}\right)=V\left(H_{2}\right)=\left\{v_{1}, v_{2}, v_{3}, v_{4}\right\}$ and $A\left(H_{1}\right)=\left\{\left(v_{1}, v_{2}\right),\left(v_{2}, v_{1}\right),\left(v_{1}, v_{4}\right),\left(v_{3}, v_{2}\right),\left(v_{3}, v_{1}\right),\left(v_{4}, v_{3}\right),\left(v_{2}, v_{4}\right)\right\}$, and $A\left(H_{2}\right)=\left\{\left(v_{1}, v_{3}\right),\left(v_{1}, v_{4}\right)\right.$, $\left.\left(v_{4}, v_{3}\right),\left(v_{3}, v_{4}\right),\left(v_{3}, v_{2}\right),\left(v_{4}, v_{2}\right),\left(v_{2}, v_{1}\right)\right\}$. Then we have these observations.

(i) For $i \in\{1,2\}, H_{i}$ are supereulerians .

(ii) Let $H_{1}$ and $H_{2}$ have spanning closed ditrails $S_{1}$ and $S_{2}$ respectively, and $a_{1}=\left(v_{4}, v_{3}\right) \in A\left(S_{1}\right)$ 
and $a_{2}=\left(v_{2}, v_{1}\right) \in A\left(S_{2}\right)$.

(iii) For $i \in\{1,2\}, a_{i} \in A\left(S_{i}\right)$ as depicted in Figure 3.

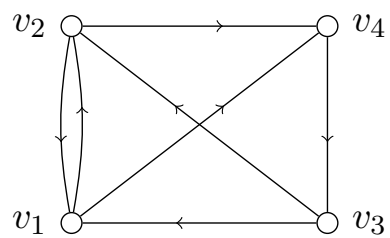

$H_{1}$

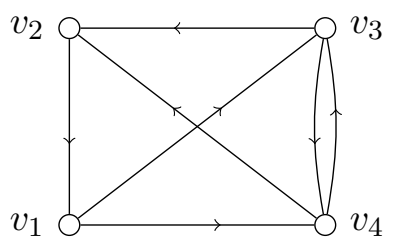

$\mathrm{H}_{2}$

Figure 3: $H_{1}$ and $H_{2}$.

(2) We will present $\mathrm{H}_{1} \oplus_{2} \mathrm{H}_{2}$ digraph, and then we will use the $\mathrm{H}_{1} \oplus_{2} \mathrm{H}_{2}$ digraph to built an infinite family of digraphs to show that if condition (i) in Proposition 4.3.1 does not hold then any 2 sum with the $\mathrm{H}_{1} \oplus_{2} \mathrm{H}_{2}$ digraph will may not supereulerian.

(i) Let $V\left(H_{1} \oplus_{2} H_{2}\right)=\left\{v_{1}, v_{2}, v_{3}, v_{4}, v_{5}, v_{6}\right\}$ and $A\left(H_{1} \oplus_{2} H_{2}\right)=\left\{\left(v_{1}, v_{2}\right),\left(v_{2}, v_{1}\right),\left(v_{1}, v_{4}\right),\left(v_{2}, v_{3}\right)\right.$, $\left.\left(v_{2}, v_{4}\right),\left(v_{3}, v_{1}\right),\left(v_{4}, v_{3}\right),\left(v_{3}, v_{6}\right),\left(v_{3}, v_{5}\right),\left(v_{5}, v_{4}\right),\left(v_{5}, v_{6}\right),\left(v_{6}, v_{5}\right),\left(v_{6}, v_{4}\right)\right\}$. Then we observe $H_{1} \oplus_{2}$ $H_{2}$ may not be supereulerian since $a=\left(v_{4}, v_{3}\right) \in A\left(S_{i}\right)$ for $i=\{1,2\}$ where $v_{2}=v_{4}$ and $v_{1}=v_{3}$, as depicted in Figure 4

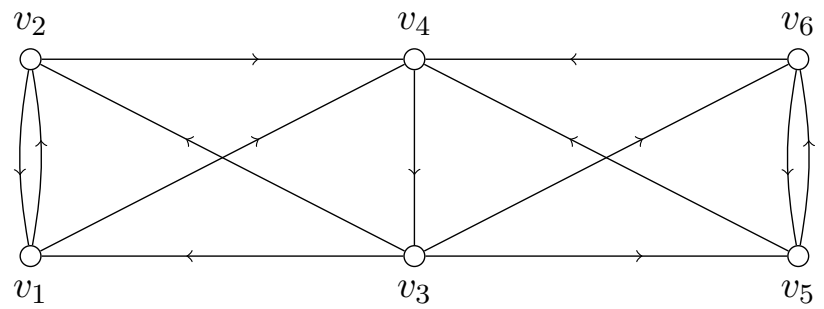

Figure 4: $\mathrm{H}_{1} \oplus_{2} \mathrm{H}_{2}$.

(ii) Using the notation in Figure 3, we can fix the vertex set of one of the two $K_{2}^{*}$ 's in $H_{1} \oplus_{2} H_{2}, H_{1} \oplus_{2} H_{2}\left[\left\{v_{1}, v_{2}\right\}\right]$ and $H_{1} \oplus_{2} H_{2}\left[\left\{v_{5}, v_{6}\right\}\right]$, and define it as a distinguished pair. Let $\{x, y\}$ be a distinguished pair of $H_{1} \oplus_{2} H_{2}$, and denote $H_{1} \oplus_{2} H_{2}$ by $D(x, y):=H_{1} \oplus_{2} H_{2}(x, y)$. Let $D_{1}\left(x^{\prime}, y^{\prime}\right)$ and $D_{2}\left(x^{\prime \prime}, y^{\prime \prime}\right)$ be the two copies of $D\left(v_{1}, v_{2}\right)$. Obtain a new digraph $D_{1} \oplus_{2} D_{2}$ from $D_{1}$ and $D_{2}$ by identifying $x^{\prime}$ and $x^{\prime \prime}$ to form a new vertex $x$, and identifying $y^{\prime}$ and $y^{\prime \prime}$ to form a new vertex $y$. See figure 5 for an drawing of $D_{1} \oplus_{2} D_{2}$ with $x=v_{1}$ and $y=v_{2}$. 


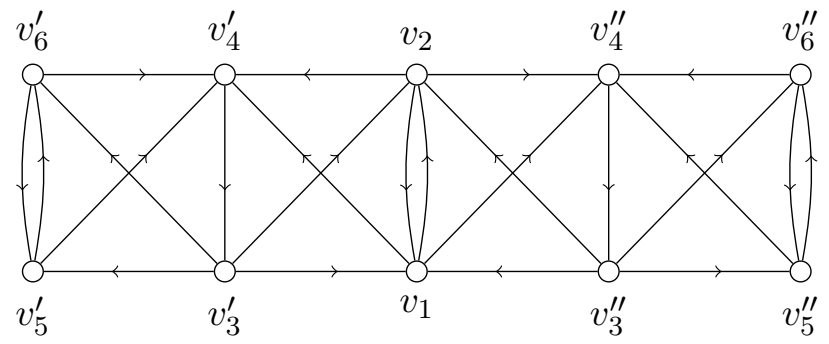

Figure 5: $D_{1} \oplus_{2} D_{2}$.

Theorem 4.3.3. [36] If a strict digraph on $n \geq 3$ vertices has $(n-1)^{2}+1$ or more arcs, then it is hamiltonian-connected.

Corollary 4.3.4. Let $D_{1}$ be a strict digraph on $n_{1} \geq 3$ vertices and with $\left|A\left(D_{1}\right)\right| \geq\left(n_{1}-1\right)^{2}+1$. If $D_{2}$ is a supereulerian digraph, then $D_{1} \oplus_{2} D_{2}$ is supereulerian.

Proof. By Theorem 4.3.3, $D_{1}$ is hamiltonian-connected. Then by Proposition 4.3.1(ii), $D_{1} \oplus_{2} D_{2}$ is supereulerian.

Two classes of supereulerain digraphs seem to be of particular interests in studying supereulerian digraph 2-sums. We first present their definitions.

Definition 4.3.5. Let $D$ be a digraph such that either $D=K_{1}$ or $A(D) \neq \emptyset$. If for any $u, v \in V(D), D$ contains a symmetric dipath from $u$ to $v$, then $D$ is called a symmetrically connected digraph.

Given a digraph $D$, define a relation $\sim$ on $V(D)$ such that $u \sim v$ if and only if $u=v$ or $D$ has a symmetrically connected subdigraph $H$ with $u, v \in V(H)$. By definition, one can routinely verify that $\sim$ is an equivalence relation. Each equivalence class induces a symmetrically connected component of $D$. Hence $D$ is symmetrically connected if and only if $D$ has only one symmetrically connected component. A symmetrically connected component of $D$ is also called a maximal symmetrically connected subdigraph of $D$. When $D$ has more than one symmetrically connected components, we have the following definition.

Definition 4.3.6. Let $D$ be a weakly connected digraph and $\left\{H_{1}, H_{2}, \ldots, H_{c}\right\}$ be the set of maximal symmetrically connected subdigraphs of $D$ with $c \geq 2$. If for any proper nonempty subset $\mathcal{J} \subset\left\{H_{1}, H_{2}, \ldots, H_{c}\right\}$,

there exist an $H_{i} \in \mathcal{J}$, a vertex $v \in V\left(H_{i}\right)$, and an $H_{j} \notin \mathcal{J}$ such that $N_{D}^{+}(v) \cap V\left(H_{j}\right) \neq \emptyset$ and $N_{D}^{-}(v) \cap V\left(H_{j}\right) \neq \emptyset$, 
then $D$ is partially symmetric.

It is known that both symmetrically connected digraphs and partially symmetric digraphs are supereulerian.

Theorem 4.3.7. ( [28]) Each of the following holds.

(i) Every symmetrically connected digraph is supereulerian.

(ii) Every partially symmetric digraph is supereulerian.

A main result of this section is to show that the digraph 2-sums of symmetrically connected or partially symmetric digraphs are supereulerian.

Lemma 4.3.8. Let $D_{1}$ and $D_{2}$ be two vertex disjoint digraphs with $a_{1}=\left(v_{11}, v_{12}\right) \in A\left(D_{1}\right)$ and $a_{2}=\left(v_{21}, v_{22}\right) \in A\left(D_{2}\right)$, and let $D_{1} \oplus_{2} D_{2}$ denote $D_{1} \oplus_{a_{1}, a_{2}} D_{2}$. Each of the following holds.

(i) If $D_{1}$ and $D_{2}$ are symmetrically connected, then $D_{1} \oplus_{2} D_{2}$ is symmetrically connected.

(ii) If $D_{1}$ and $D_{2}$ are partially symmetric, then $D_{1} \oplus_{2} D_{2}$ is partially symmetric.

(iii) If $D_{1}$ is symmetric and $D_{2}$ is partially symmetric, then $D_{1} \oplus_{2} D_{2}$ is partially symmetric.

Proof. (i) For any vertices $x, y \in V\left(D_{1} \oplus_{2} D_{2}\right)$, we shall show that $D_{1} \oplus_{2} D_{2}$ always has a symmetric $(x, y)$-dipath. If for some $i \in\{1,2\}$, we have $x, y \in V\left(D_{i}\right)$, then as $D_{i}$ is symmetrically connected, $D_{i}$ contains a symmetric $(x, y)$-dipath $P$. Since $D_{i}$ is a subdigraph of $D_{1} \oplus_{2} D_{2}$, $P$ is also a symmetric $(x, y)$-dipath of $D_{1} \oplus_{2} D_{2}$. Hence we may assume that $x \in V\left(D_{1}\right)$ and $y \in V\left(D_{2}\right)$. Since $D_{1}$ and $D_{2}$ are symmetrically connected, $D_{1}$ contains a symmetric $\left(x, v_{11}\right)$ dipath $P_{1}$ and $D_{2}$ contains a symmetric $\left(v_{21}, y\right)$-dipath $P_{2}$. By Definition 4.2.1, $v_{11}$ and $v_{21}$ represent the same vertex in $D_{1} \oplus_{2} D_{2}$, and so $D_{1} \oplus_{2} D_{2}\left[A\left(P_{1}\right) \cup A\left(P_{2}\right)\right]$ is a symmetric $(x, y)$ dipath in $D_{1} \oplus_{2} D_{2}$.

(ii) Fix $i \in\{1,2\}$. Since $D_{i}$ is partially symmetric, for some integer $c_{i}>1$, let $\left\{H_{i 1}^{\prime}, H_{i 2}^{\prime}, \ldots, H_{i c_{i}}^{\prime}\right\}$ be the set of all maximal symmetrically connected subdigraphs of $D_{i}$. Without loss of generality, we assume that $v_{11} \in V\left(H_{11}^{\prime}\right)$ and $v_{21} \in V\left(H_{21}^{\prime}\right)$; and for some $s, t$ with $1 \leq s \leq c_{1}$ and $1 \leq t \leq c_{2}, v_{12} \in V\left(H_{1 s}^{\prime}\right)$ and $v_{22} \in V\left(H_{2 t}^{\prime}\right)$. (We allow the possibility that $s=1$ and/or $t=1$ ). Define, for $1 \leq h \leq c_{1}$ and $1 \leq j \leq c_{2}$,

$$
H_{1 h}=\left\{\begin{array}{ll}
H_{1 h}^{\prime} & \text { if } h \notin\{1, s\} \\
H_{11}^{\prime} \cup H_{21}^{\prime} & \text { if } h=1 \\
H_{1 s}^{\prime} \cup H_{2 t}^{\prime} & \text { if } h=s
\end{array} \quad \text { and } H_{2 j}= \begin{cases}H_{2 j}^{\prime} & \text { if } j \notin\{1, t\} \\
H_{11}^{\prime} \cup H_{21}^{\prime} & \text { if } j=1 \\
H_{1 s}^{\prime} \cup H_{2 t}^{\prime} & \text { if } j=t\end{cases}\right.
$$


Then $\mathcal{H}=\left\{H_{11}, H_{12}, \ldots, H_{1 c_{1}}, H_{21}, H_{22}, \ldots, H_{2 c_{2}}\right\}$ is the set of all maximal symmetrically connected subdigraphs of $D_{1} \oplus_{2} D_{2}$. Note that $H_{11}=H_{21}$ and $H_{1 s}=H_{2 t}$. We shall show by definition that $D_{1} \oplus_{2} D_{2}$ is partially symmetric. To do that, let $\mathcal{J}$ be a nonempty proper subset of $\mathcal{H}$. We shall show that (4.2) holds.

Since $\mathcal{H}=\left\{H_{11}, H_{12}, \ldots, H_{1 c_{1}}, H_{21}, H_{22}, \ldots, H_{2 c_{2}}\right\}$, we either have $\mathcal{J} \cap\left\{H_{11}, H_{12}, \ldots, H_{1 c_{1}}\right\} \neq$ $\emptyset$ or $\mathcal{J} \cap\left\{H_{21}, H_{22}, \ldots, H_{2 c_{2}}\right\} \neq \emptyset$. By symmetry, we may assume that $\mathcal{J} \cap\left\{H_{11}, H_{12}, \ldots, H_{1 c_{1}}\right\} \neq$ $\emptyset$.

Suppose first that $\left\{H_{11}, H_{12}, \ldots, H_{1 c_{1}}\right\}-\mathcal{J} \neq \emptyset$. Let $\mathcal{J}^{\prime}=\left\{H_{1 h}^{\prime} \mid H_{1 h} \in \mathcal{J}\right\}$. Then $\left\{H_{11}^{\prime}, H_{12}^{\prime}\right.$, $\left.\ldots, H_{1 c_{1}}^{\prime}\right\}-\mathcal{J}^{\prime} \neq \emptyset$. Since $D_{1}$ is partially symmetric, there exist an $H_{1 h_{0}}^{\prime} \in \mathcal{J}^{\prime}$, a vertex $v \in V\left(H_{1 h_{0}}^{\prime}\right)$, and an $H_{1 j_{0}}^{\prime} \in\left\{H_{11}^{\prime}, H_{12}^{\prime}, \ldots, H_{1 c_{1}}^{\prime}\right\}-\mathcal{J}^{\prime}$ such that

$$
N_{D_{1}}^{+}(v) \cap V\left(H_{1 j_{0}}^{\prime}\right) \neq \emptyset \text { and } N_{D_{1}}^{-}(v) \cap V\left(H_{1 j_{0}}^{\prime}\right) \neq \emptyset .
$$

This implies that the vertex $v \in V\left(H_{1 h_{0}}\right), H_{1 h_{0}} \in \mathcal{J}$, and $H_{1 j_{0}} \notin \mathcal{J}$ such that

$$
N_{D_{1} \oplus_{2} D_{2}}^{+}(v) \cap V\left(H_{1 j_{0}}\right) \neq \emptyset \text { and } N_{D_{1} \oplus_{2} D_{2}}^{-}(v) \cap V\left(H_{1 j_{0}}\right) \neq \emptyset .
$$

Thus (4.2) holds in this case.

Hence we may assume that $\left\{H_{11}, H_{12}, \ldots, H_{1 c_{1}}\right\} \subset \mathcal{J}$. Since $\mathcal{J}$ is a proper subset, we must have $\left\{H_{21}, H_{22}, \ldots, H_{2 c_{2}}\right\}-\mathcal{J} \neq \emptyset$. Since $H_{21}=H_{11} \in \mathcal{J}$, we also have $\left\{H_{21}, H_{22}, \ldots, H_{2 c_{2}}\right\} \cap$ $\mathcal{J} \neq \emptyset$. With a similar argument, we conclude that (4.2) must also hold in this case.

(iii) Let $H_{0}=D_{1}$ and let $\left\{H_{1}^{\prime}, H_{2}^{\prime}, \ldots, H_{c}^{\prime}\right\}$ be the set of all maximal symmetrically connected subdigraphs of $D_{2}$ with $v_{21} \in V\left(H_{1}^{\prime}\right)$ and for some $j \in\{1,2, \ldots, c\}, v_{22} \in V\left(H_{j}^{\prime}\right)$. (We allow the possibility that $j=1$ ). Define

$$
H_{i}=\left\{\begin{array}{ll}
H_{1}^{\prime} \cup H_{0} \cup H_{j}^{\prime} & \text { if } i=1 \text { or } i=j \\
H_{i}^{\prime} & \text { if } i \notin\{1, j\}
\end{array} .\right.
$$

Then $\mathcal{H}=\left\{H_{1}, H_{2}, \ldots, H_{c}\right\}$ is the set of all maximal symmetrically connected subdigraphs of $D_{1} \oplus_{2} D_{2}$. Note that $H_{1}=H_{j}$ with this notation. Let $\mathcal{J}$ be a nonempty proper subset of $\mathcal{H}$. We shall show that (4.2) holds.

Let $J^{\prime}=\left\{H_{i}^{\prime} \mid H_{i} \in \mathcal{J}\right\}$. Since $\mathcal{J}$ is proper, $\mathcal{J}^{\prime}$ is a nonempty proper subset of $\left\{H_{1}^{\prime}, H_{2}^{\prime}, \ldots, H_{c}^{\prime}\right\}$. Since $D_{2}$ is partially symmetric, by Definition 4.3.6, there exist an $H_{i_{0}}^{\prime} \in \mathcal{J}^{\prime}$, a vertex $v \in V\left(H_{i_{0}}^{\prime}\right)$, and an $H_{j_{0}}^{\prime} \in\left\{H_{1}^{\prime}, H_{2}^{\prime}, \ldots, H_{c}^{\prime}\right\}-\mathcal{J}^{\prime}$ such that

$$
N_{D_{1}}^{+}(v) \cap V\left(H_{j_{0}}^{\prime}\right) \neq \emptyset \text { and } N_{D_{1}}^{-}(v) \cap V\left(H_{j_{0}}^{\prime}\right) \neq \emptyset
$$


This implies that vertex $v \in V\left(H_{i_{0}}\right), H_{i_{0}} \in \mathcal{J}$ and $H_{j_{0}} \notin \mathcal{J}$ such that

$$
N_{D_{1} \oplus_{2} D_{2}}^{+}(v) \cap V\left(H_{j_{0}}\right) \neq \emptyset \text { and } N_{D_{1} \oplus_{2} D_{2}}^{-}(v) \cap V\left(H_{j_{0}}\right) \neq \emptyset .
$$

Thus (4.2) holds, and so by definition, $D_{1} \oplus_{2} D_{2}$ is partially symmetric.

Theorem 4.3.9. Let $D_{1}$ and $D_{2}$ be two digraphs. Each of the following holds.

(i) If $D_{1}$ and $D_{2}$ are symmetrically connected, then $D_{1} \oplus_{2} D_{2}$ is supereulerian.

(ii) If $D_{1}$ and $D_{2}$ are partially symmetric, then $D_{1} \oplus_{2} D_{2}$ is supereulerian.

(iii) If $D_{1}$ is symmetric and $D_{2}$ is partially symmetric, then $D_{1} \oplus_{2} D_{2}$ is supereulerian.

Proof. This follows from Theorem 4.3.7 and Lemma 4.3.8.

If the absence of condition (ii) in Theorem 4.3.9 is not sufficient to guarantee that $D_{1} \oplus_{2} D_{2}$ will be a supereulerian. We have introduce the following counterexample.

Example 4.3.10. (1)Let $D_{1}$ be a digraph with $V\left(D_{1}\right)=\left\{v_{1}, v_{2}, v_{3}, v_{4}\right\}$ and $A\left(D_{1}\right)=\left\{\left(v_{1}, v_{2}\right),\left(v_{2}, v_{1}\right)\right.$, $\left.\left(v_{1}, v_{4}\right),\left(v_{2}, v_{3}\right),\left(v_{3}, v_{4}\right),\left(v_{4}, v_{3}\right),\left(v_{3}, v_{1}\right),\left(v_{4}, v_{2}\right)\right\}$, and let $D_{2}$ be a digraph with $V\left(D_{2}\right)=\left\{v_{1}, v_{2}, v_{3}\right.$, $\left.v_{4}, v_{5}, v_{6}\right\}$ and $A\left(D_{2}\right)=\left\{\left(v_{1}, v_{2}\right),\left(v_{2}, v_{1}\right),\left(v_{2}, v_{4}\right),\left(v_{2}, v_{3}\right),\left(v_{3}, v_{4}\right),\left(v_{4}, v_{3}\right),\left(v_{4}, v_{1}\right),\left(v_{3}, v_{1}\right),\left(v_{5}, v_{4}\right)\right.$, $\left.\left(v_{5}, v_{6}\right),\left(v_{6}, v_{5}\right),\left(v_{6}, v_{4}\right),\left(v_{3}, v_{6}\right),\left(v_{3}, v_{5}\right)\right\}$, as depicted in Figure 6 . Then we have these observations.

(i) For $i \in\{1,2\}, D_{i}$ does not have a symmetric $\left(v_{3}, v_{2}\right)$-dipath, and so $D_{i}$ is not symmetrically connected.

(ii) For $i \in\{1,2\}$, the maximal symmetrically connected subdigraph of $D_{i}$ are $H_{1}=D_{i}\left[\left\{v_{1}, v_{2}\right\}\right]$ , $H_{2}=D_{i}\left[\left\{v_{3}, v_{4}\right\}\right]$ and $H_{3}=D_{2}\left[\left\{v_{5}, v_{6}\right\}\right]$.

(iii) By Definition 4.3.6(i), $D_{1}$ is partially symmetric but $D_{2}$ is not partially symmetric.
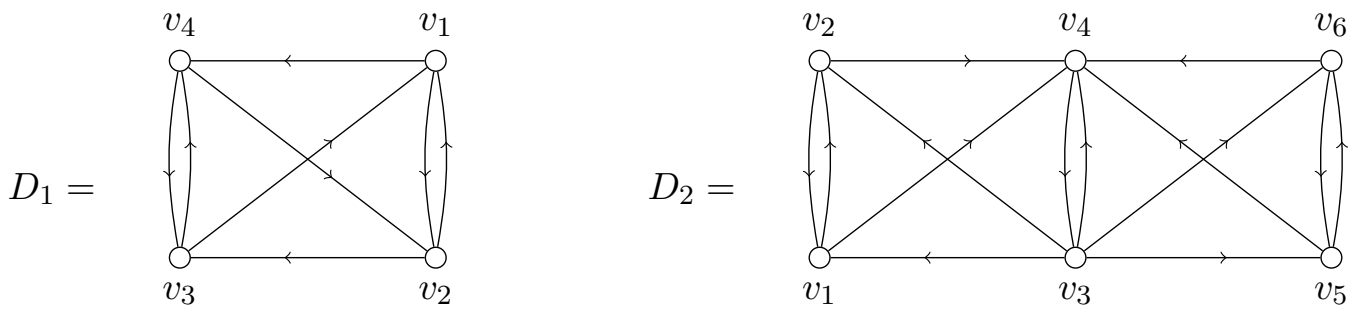

Figure 6. The digraphs $D_{1}$ and $D_{2}$.

(2) We will present $D_{1} \oplus_{2} D_{2}$ digraph, and then we will use the $D_{1} \oplus_{2} D_{2}$ digraph to built an infinite family of digraphs to show that the condition (ii) in Therorem 4.3 .9 in some sense is 
the best possible.

(i) Let $V\left(D_{1} \oplus_{2} D_{2}\right)=\left\{v_{1}, v_{2}, v_{3}, v_{4}, v_{5}, v_{6}, v_{7}, v_{8}\right\}$ and $A\left(D_{1} \oplus_{2} D_{2}\right)=\left\{\left(v_{1}, v_{2}\right),\left(v_{2}, v_{1}\right),\left(v_{1}, v_{4}\right),\left(v_{2}, v_{3}\right)\right.$, $\left(v_{3}, v_{4}\right),\left(v_{4}, v_{3}\right),\left(v_{3}, v_{1}\right),\left(v_{4}, v_{2}\right)\left(v_{5}, v_{6}\right),\left(v_{6}, v_{5}\right),\left(v_{4}, v_{5}\right),\left(v_{3}, v_{5}\right),\left(v_{6}, v_{4}\right),\left(v_{6}, v_{3}\right),\left(v_{6}, v_{7}\right),\left(v_{6}, v_{8}\right),\left(v_{8}, v_{5}\right)$, $\left.\left(v_{7}, v_{5}\right),\left(v_{7}, v_{8}\right),\left(v_{8}, v_{7}\right)\right\}$, as depicted in Figure 7

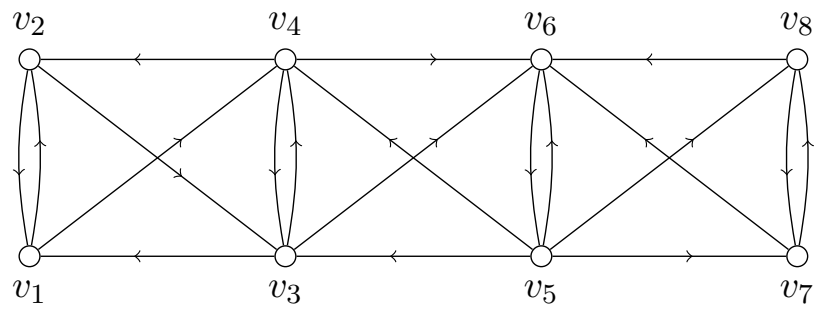

Figure 7: $D_{1} \oplus_{2} D_{2}$ may not be a supereulerian.

(ii) Using the notation in Figure 13, we can fix the vertex set of one of the two $K_{2}^{*}$ 's in $D_{1} \oplus_{2} D_{2}, D_{1} \oplus_{2} D_{2}\left[\left\{v_{1}, v_{2}\right\}\right]$, and $D_{1} \oplus_{2} D_{2}\left[\left\{v_{7}, v_{8}\right\}\right]$, and define it as a distinguished pair. Let $\{a, b\}$ be a distinguished pair of $D_{1} \oplus_{2} D_{2}$, and denote $D_{1} \oplus_{2} D_{2}$ by $Y(a, b):=D_{1} \oplus_{2} D_{2}(a, b)$. Let $Y_{1}\left(a^{\prime}, b^{\prime}\right)$ and $Y_{2}\left(a^{\prime \prime}, b^{\prime \prime}\right)$ be the two copies of $Y\left(v_{1}, v_{2}\right)$. Obtain a new digraph $Y_{1} \oplus_{2} Y_{2}$ from $Y_{1}$ and $Y_{2}$ by identifying $a^{\prime}$ and $a^{\prime \prime}$ to form a new vertex $a$, and identifying $b^{\prime}$ and $b^{\prime \prime}$ to form a new vertex $b$. See figure 8 for an drawing of $Y_{1} \oplus_{2} Y_{2}$ with $a=v_{1}$ and $b=v_{2}$.

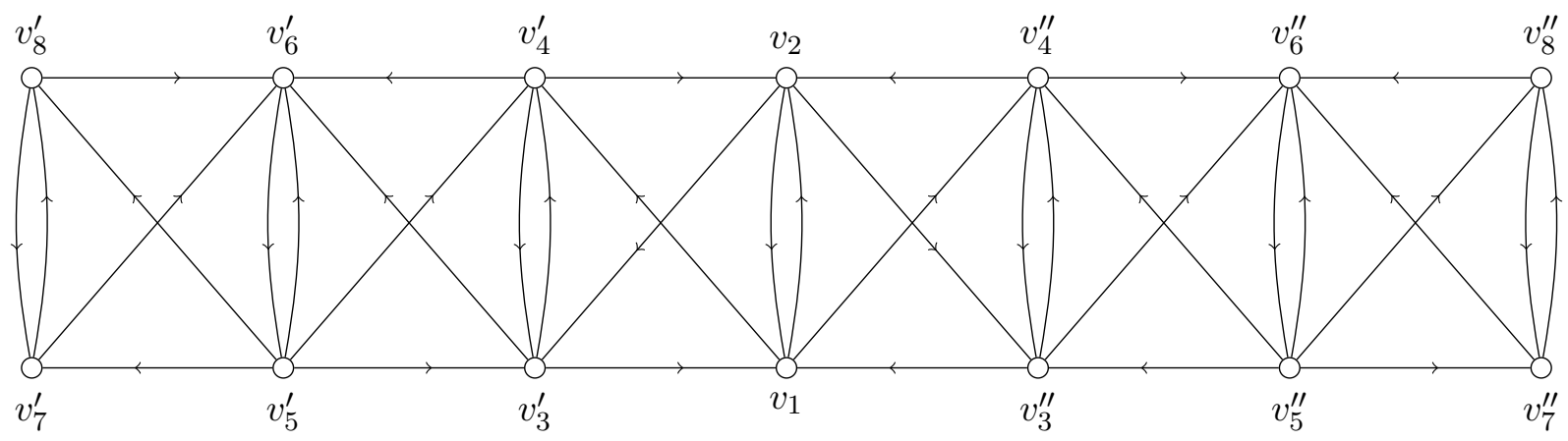

Figure 8: $Y_{1} \oplus_{2} Y_{2}$.

It is also natural to consider sufficient conditions on $D_{1}$ and $D_{2}$ for $D_{1} \oplus_{2} D_{2}$ to be hamiltonian.

Theorem 4.3.11. If $D_{1}$ is hamiltonian and $D_{2}$ is hamiltonian-connected digraphs, then $D_{1} \oplus_{2}$ $D_{2}$ is hamiltonian. 
Proof. Let $V\left(D_{1}\right)=\left\{v_{11}, v_{12}, \ldots, v_{1 n_{1}}\right\}$ with $C=v_{11} v_{12} \ldots v_{1 n_{1}} v_{11}$ be a hamiltonian dicycle of $D_{1}$ and $V\left(D_{2}\right)=\left\{v_{21}, v_{22}, \ldots, v_{2 n_{2}}\right\}$. Let $a_{1}=\left(v_{11}, v_{12}\right) \in A\left(D_{1}\right)$ and $a_{2}=\left(v_{21}, v_{22}\right) \in A\left(D_{2}\right)$, and $D_{1} \oplus_{2} D_{2}=D_{1} \oplus_{a_{1}, a_{2}} D_{2}$. Since $D_{2}$ is hamiltonian-connected, $D_{2}$ contains a $\left(v_{21}, v_{22}\right)$ hamiltonian dipath $P$. Thus $\left(C-\left\{a_{1}\right\}\right) \cup P$ is a hamiltonian dicycle in $D_{1} \oplus_{2} D_{2}$.

Theorem 4.3.12. [37] If a semicomplete digraph $D$ is 4-strong, then $D$ is hamiltonianconnected.

By Theorem 4.3.11 and 4.3.12, we have the following corollary.

Corollary 4.3.13. Let $D_{1}$ and $D_{2}$ be two 4-strong semicomplete digraphs, then $D_{1} \oplus_{2} D_{2}$ is hamiltonian. 


\section{Bibliography}

[1] J. A. Bondy and U. S. R. Murty, Graph Theory, Springer, New York, 2008.

[2] J. Bang-Jensen and G. Gutin, Digraphs: Theory, Algorithms and Applications Second Edition, Springer, (2010)

[3] D. W. Barnette, Cycle covers of planar 3-connected graphs, J. Combin. Math. Combin. Comput., 20 (1996), 245-253.

[4] J. A. Bondy, Small cycle double cover of graphs. Cycles and Rays. Kluwer Academic Publishers, Dordecht (1990) 21-40.

[5] P. Camion, Chemins et circuits hamiltoniens des graphes complets, C. R. Acad.Sci. Paris, 249 (1959) 2151-2152.

[6] G. Fan, Subgraph covering and edge switchings, J. Combin. Theory, Ser. B, (84) (2002) 54-83.

[7] J. W. Moon, On subtournaments of a tournament, Can. Math. Bull., 9 (1966) 297-301.

[8] Robert J. Douglas, Tournaments that admit exactly one Hamiltonian circuit, 30 June 1969.

[9] H.-J. Lai, Cycle covers of planar graphs, Congressus Numerantium, 122 (1996) 33-46.

[10] H.-J. Lai and H. Y. Lai, Cycle covering of plane triangulations, J. Combin. Math. Combin. Comput., 10 (1991) 3-21.

[11] H.-J. Lai and H. Y. Lai, Cycle covers in graphs without subdivisions of $K_{4}$, J. Combin. Math. Combin. Comput., 9 (1991), 201-207.

[12] H.-J. Lai and H. Y. Lai, Small cycle covers of planar graphs, Congressus Numerantium, 85 (1991) 203-209.

[13] H.-J. Lai and X. Li, Small cycle cover of 2-connected cubic graphs, Discrete Math., 269 (2003), 295-302. 
[14] Y. X. Luo and R. S. Chen, Cycle covers of 2-connected 3-regular graphs, (Chinese, Chinese summary), Math. Appl., 9 (1996), suppl., 23-25.

[15] F. Yang and X. Li, Small cycle cover of 3-connected cubic graphs, Discrete Math., 311 (2011) 186-196.

[16] J. Bang-Jensen and J. Huang, Quasi-transitive digraphs, J Graph Theory 20(2) (1995), 141-161.

[17] J. Bang-Jensen and A. Maddaloni, Sufficient conditions for a digraph to be supereulerian, J. Graph Theory 79(1) (2015) 8-20.

[18] F. T. Boesch, C. Suffel, and R. Tindell, The spanning subgraphs of eulerian graphs, J. Graph Theory 1 (1977) 79-84.

[19] P. A. Catlin, A reduction method to find spanning Eulerian subgraphs, J. Graph Theory 12 (1988) 29-44.

[20] P. A. Catlin, Supereulerian graphs: a survey, J. of Graph Theory 16 (1992) 177-196.

[21] Z. H. Chen, H.-J. Lai, Reduction techniques for super-Eulerian graphs and related topics-a survey, Combinatorics and graph theory' 95, Vol. 1 (Hefei), World Sci. Publishing, River Edge, NJ 1995 pp. 53-69.

[22] V. Chvátal and P. Erdös, A note on Hamiltonian circuits. Discrete Math., 2, (1972) 111-113.

[23] F. Jaeger, A note on subeulerian graphs, J. Graph Theory 3, (1979) 91-93.

[24] H.-J. Lai, Y. Shao, and H. Yan. An update on supereulerian Graphs, WSEAS Transactions on Mathematics 12(9) (2013) 926-940.

[25] C. Thomassen. Long cycles in digraphs. Proc. London Math. Soc. (3), 42, (1981) 231-251.

[26] W. R. Pulleyblank, A note on graphs spanned by Eulerian graphs, J. Graph Theory 3 (1979) 309-310.

[27] M. J. Algefari and H.-J. Lai, Supereulerian digraphs with large arc-strong connectivity, Journal of Graph Theory, DOI: 10.1002/jgt.21885.

[28] M. J. Algefari, K. A. Alsatami, H.-J. Lai and J. Liu, Supereulerian Digraphs with given local structures, Information Processing Letters, 116, (2016) 321-326.

[29] A. Benhochine, L. Clark, N. Köhler and H. J. Veldman, On circuits and pancyclic line graphs, Journal of Graph Theory, 10, (1986) 411-425. 
[30] F. T. Boesch, C. Suffel and R. Tindell, The spanning subgraphs of eulerian graphs, Journal of Graph Theory, 1, (1977) 79-84.

[31] G. Gutin, Cycles and paths in directed graphs, PhD thesis, School of Mathematics, Tel Aviv University, 1993.

[32] G. Gutin, Connected (g; f)-factors and supereulerian digraphs, Ars Combinatoria, 54, (2000) 311-317.

[33] L. S. Han, H.-J. Lai, L. Xiong and H. Yan, The Chvátal-Erdös condition for supereulerian graphs and the Hamiltonian index, Discrete Mathematics, 310, (2010) 2082-2090.

[34] Y. M. Hong, H.-J. Lai and Q. H. Liu, Supereulerian Digraphs, Discrete Mathematics, 330, (2014) 87-95.

[35] H.-J. Lai, Y. Shao and H. Yan, An update on supereulerian Graphs, WSEAS Transactions on Math-ematics, 12(9), (2013) 926-940.

[36] M. Lewin, On maximal circuits in directed graphs, Journal of Combinatorial Theory. Series B, 18, (1975) 175-179.

[37] C. Thomassen, Hamiltonian-connected tournaments, Journal of Combinatorial Theory. Series $\mathrm{B}, 28(2)$, (1980) 142-163. 